\title{
Affine dual equivalence and $k$-Schur functions
}

\author{
Sami H. AssaF* and Sara C. Billey ${ }^{\dagger}$
}

The $k$-Schur functions were first introduced by Lapointe, Lascoux and Morse [18] in the hopes of refining the expansion of Macdonald polynomials into Schur functions. Recently, an alternative definition for $k$-Schur functions was given by Lam, Lapointe, Morse, and Shimozono [17] as the weighted generating function of starred strong tableaux which correspond with labeled saturated chains in the Bruhat order on the affine symmetric group modulo the symmetric group. This definition has been shown to correspond to the Schubert basis for the affine Grassmannian of type $A$ [15] and at $t=1$ it is equivalent to the $k$-tableaux characterization of Lapointe and Morse [22]. In this paper, we extend Haiman's dual equivalence relation on standard Young tableaux [12] to all starred strong tableaux. The elementary equivalence relations can be interpreted as labeled edges in a graph which share many of the properties of Assaf's dual equivalence graphs. These graphs display much of the complexity of working with $k$-Schur functions and the interval structure on $\widetilde{S}_{n} / S_{n}$. We introduce the notions of flattening and squashing skew starred strong tableaux in analogy with jeu de taquin slides in order to give a method to find all isomorphism types for affine dual equivalence graphs of rank 4 . Finally, we state some open problems on other ways to generalize dual equivalence.

\section{Introduction}

Classically, the Schur functions have played a central role in the theory of symmetric functions [27]. They also appear in geometry as representatives for Schubert classes in the cohomology rings of Grassmannian manifolds, and they appear in representation theory as the Frobenius characteristics of irreducible $S_{n}$ representations and as the trace for certain irreducible $G L_{n}$ representations.

In [18], Lapointe, Lascoux, and Morse introduced a new larger family of symmetric functions which includes the Schur functions, namely the

arXiv: 1201.2128

*Supported by an NSF postdoctoral fellowship.

${ }^{\dagger}$ Supported by grants DMS-0800978 and DMS-1101017 from the NSF. 
$k$-Schur functions, with similar connections both to geometry and to representation theory. The $k$-Schur functions were defined in hopes of refining and ultimately proving the Macdonald Positivity Conjecture [26]. Precisely, Lapointe, Lascoux, and Morse conjectured that the Macdonald polynomials expand into $k$-Schur functions with polynomial coefficients in two parameters $q, t$ with nonnegative integer coefficients, and that the $k$-Schur functions expand into Schur functions with polynomial coefficients with parameter $t$ and nonnegative integer coefficients. Haiman [11] has since shown that the Macdonald polynomials are the Frobenius characteristic of a bigraded $S_{n^{-}}$ module defined by Garsia and Haiman [7] using the geometry of the Hilbert scheme of points in the plane. This resolved the $n$ ! Conjecture and provided the first proof of Macdonald positivity.

At this time, a number of conjecturally equivalent definitions for $k$-Schur functions exist $[17,18,19,20,21,22]$, making the term " $k$-Schur function" rather ambiguous. In this paper, we advocate for the geometrically inspired definition as the weighted generating function of starred strong tableaux presented by Lam, Lapointe, Morse and Shimozono [17]. This definition at $t=1$ is equivalent to the $k$-tableaux characterization in [22] which has been shown to represent the Schubert basis in the homology of the affine Grassmannian of type $A$ [15]. Furthermore, the starred strong tableaux are a natural generalization of standard tableaux which appear throughout combinatorics.

Recently, Lam, Lapointe, Morse and Shimozono proved that the $k$-Schur functions as defined below except with $t=1$ are Schur positive [16]. Their approach shows how $k$-Schur functions relate to $k+1$-Schur functions when the $t$ is not included.

It is an open problem to show that the $k$-Schur functions including the $t$ statistic are Schur positive. Toward proving this conjecture, we define a family of involutions on starred strong tableaux which generalize Haiman's elementary dual equivalence moves on standard Young tableaux [12]. Using these involutions, one can put a graph structure on starred strong tableaux which satisfies many of the same axioms as the dual equivalence graphs defined by the first author in [1]. As our model for dual equivalence is based on the poset of $n$-cores induced from Young's lattice, our results extend to $k$-Schur functions indexed by skew shapes. Our main result is that these graphs, which we call affine dual equivalence graphs, are locally Schur positive when restricted to edges of 2 adjacent colors and the spin is constant on connected components, see Definition 4.5 and Theorem 7.15. ${ }^{1}$

\footnotetext{
${ }^{1}$ Earlier, we announced the stronger result that $k$-Schur functions as defined here are Schur positive. However, we have since realized that the proof is incomplete
} 
Jeu de taquin is an important algorithm in the theory of symmetric functions related to Littlewood-Richardson coefficients. One of the properties of jeu de taquin slides is that they commute with elementary dual equivalence moves on tableaux [12, Lemma 2.3]. There is no known analog of jeu de taquin for $k$-Schur functions at this time. Such an analog would in principle be useful for multiplying $k$-Schur functions and expanding again into $k$-Schurs. One approach to finding such a jeu de taquin algorithm is to look for sliding moves which commute with affine dual equivalence moves. In Sections 7.1 and 7.2, we describe two types of collapsing moves which commute with affine dual equivalence in specified cases. These collapsing moves are the analogs of removing empty rows and columns in a skew tableau via jeu de taquin.

One of the main consequences of our results is a connection between $k$-Schur functions and LLT polynomials which is realized by comparing the graphs for the two functions. We expect that a better understanding of these connections will ultimately show that an LLT polynomial expands into $k$ Schur functions with coefficients that are polynomials in $t$ with nonnegative integer coefficients for an appropriate value of $k$. Given Haglund's formula expanding Macdonald polynomials positively into certain LLT polynomials $[9,10]$, this would also establish the missing connection between Macdonald polynomials and $k$-Schur functions.

The outline of the paper goes as follows. In Section 2, we review the basic vocabulary on partitions, the affine symmetric group, symmetric functions and quasisymmetric functions. In particular, we review an interesting order preserving bijection between a quotient of the affine symmetric group with the $n$-core partitions relating Bruhat order to a subposet of Young's lattice. In Section 3, one definition of $k$-Schur functions expanded into fundamental quasisymmetric functions is given following [17, Conjecture 9.11]. These functions can be indexed by $n$-cores, minimal length coset representatives for $\widetilde{\mathcal{S}}_{n} / \mathcal{S}_{n}$, or $k=n-1$ bounded partitions since all three sets are in bijection. In Section 4, we review dual equivalence on standard Young tableaux along with the associated graph structures and axioms. In Section 5, we carefully study the covering relations and the rank two intervals in the poset on $n$ core partitions. In Section 6, we define the affine analog of dual equivalence operations and prove the key result that these maps are involutions which preserve the spin statistic. In Section 7, we use these maps to define the

for two reasons. First, the proof outline requires one to identify all isomorphism types for 3-colored components in affine dual equivalence graphs. Our computer verification relies on a halting problem which has not terminated. Second, the axiom (4') required in [1] is not known to hold for affine dual equivalence graphs. 
affine dual equivalence graph on starred strong tableaux of a given shape. In particular, Theorem 7.15 spells out some of the useful properties of these graphs. In Section 8, we mention some open problems on other generalizations of dual equivalence graphs. Finally, in the Appendix, we have included some examples of $k$-Schur functions expanded both in quasisymmetric functions and Schur functions along with their affine dual equivalence graphs.

\section{Basic definitions and notations}

\subsection{Partitions}

A partition $\lambda$ is a weakly decreasing sequence of non-negative integers

$$
\lambda=\left(\lambda_{1}, \lambda_{2}, \ldots, \lambda_{l}\right), \quad \lambda_{1} \geq \lambda_{2} \geq \cdots \geq \lambda_{l}>0 .
$$

The Young diagram of a partition $\lambda$ is the set of points $(i, j)$ in $\mathbb{N} \times \mathbb{N}$ such that $1 \leq i \leq \lambda_{j}$. We draw the diagram so that each point $(i, j)$ is represented by the unit cell southwest of the point. Abusing notation, we will write $\lambda$ for both the partition and its diagram. For example, the diagram of $(4,3,1)$ is

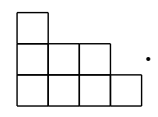

We may also represent $\lambda$ by an infinite binary string as follows. Consider the diagram of $\lambda$ lying in the $\mathbb{N} \times \mathbb{N}$ plane with infinite positive axes. Walk in unit steps along the boundary of $\lambda$, writing 1 for each vertical step and 0 for each horizontal step. For example, $(4,3,1)$ becomes

$$
\cdots 1110100101000 \cdots \text {. }
$$

Note that this establishes a bijective correspondence between partitions and doubly infinite binary strings $s$ such that $s_{i}=1$ for all $i<l$ and $s_{i}=0$ for all $i>r$ for some $l, r \in \mathbb{Z}$.

For partitions $\lambda$, $\mu$, we write $\mu \subset \lambda$ whenever the diagram of $\mu$ is contained within the diagram of $\lambda$; equivalently $\mu_{i} \leq \lambda_{i}$ for all $i$. Young's lattice is defined by the partial ordering on partitions given by containment.

A standard Young tableau of shape $\lambda$ is a saturated chain in Young's lattice from the empty partition to $\lambda$. As moving from rank $i-1$ to rank $i$ adds a single box, filling this added box with the letter $i$ uniquely records the chosen chain. Therefore standard Young tableaux are also characterized as 
bijective fillings of the cells of $\lambda$ with the letters 1 to $m$ so that entries increase along rows and up columns. Let $\operatorname{SYT}(\lambda)$ denote the set of all standard Young tableaux of shape $\lambda$, and let SYT denote the union of all $\operatorname{SYT}(\lambda)$. For example, two standard tableau of shape $(4,3,1)$ are

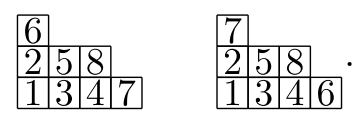

When $\mu \subset \lambda$, we may define the skew diagram $\lambda / \mu$ to be the set theoretic difference $\lambda-\mu$. A standard tableau of skew shape $\lambda / \mu$ is a saturated chain in Young's lattice from $\mu$ to $\lambda$, or, equivalently, a bijective filling of the cells of $\lambda / \mu$ with entries 1 to $m$ so that entries increase along rows and up columns.

An addable cell for a partition $\lambda$ is any cell $c$ such that $c \cup \lambda$ is again a Young diagram of a partition. Similarly, a removable cell for a partition $\lambda$ is any cell $c$ such that $\lambda-c$ is again a Young diagram of a partition.

A connected skew diagram is one where exactly one cell has no cell immediately north or west of it, and exactly one cell has no cell immediately south or east of it. Two distinct connected components can meet at one point but not along an edge of a cell. A connected skew diagram is necessarily nonempty. A ribbon is a connected skew diagram containing no $2 \times 2$ subdiagram. We may define addable and removable ribbons of $\lambda$ just as with cells; namely, a ribbon $R$ is an addable (resp. removable) ribbon for a partition $\lambda$ if $\lambda \cup R$ (resp. $\lambda-R$ ) is again a partition.

To each cell $x$ of a diagram $\lambda$ associate the content of $x$ defined by $c(x)=i-j$ where the cell $x$ lies in row $j$ and column $i$. We also consider the residue of $x$, defined as the content of $x$ modulo $n$. The content and residue of ribbons are defined with respect to the southeasternmost cell. The head of a ribbon is its southeasternmost cell, and the tail of a ribbon is its northwesternmost cell.

The hook length of $x$ is the number of squares above and to the right of $x$ in $\lambda$ including $x$ itself. Define the bandwidth of a partition to be the number of distinct contents occupied by its cells. Equivalently, the bandwidth of a non-skew partition is its maximum hook length.

An $n$-core is a partition having no removable ribbon of length $n$. Equivalently, no hook length of $\lambda$ is divisible by $n$. Young's lattice restricted to $n$-cores gives another ranked partial order, but it is not a lattice. This partial order on $n$-cores is central to the definition of $k$-Schur functions and strong tableaux given in Section 3. 


\subsection{Affine permutations}

Here we briefly recall the necessary vocabulary on affine permutations. For a more thorough treatment of the combinatorial aspects of Coxeter groups we recommend [5], specifically see Section 8.3 for details on the affine symmetric group. Recent developments on core partitions and connections to affine Weyl groups can be found in $[4,13]$.

Given $n$, consider the set $\widetilde{\mathcal{S}}_{n}$ of all bijections $w: \mathbb{Z} \longrightarrow \mathbb{Z}$ such that

$$
w(i+n)=w(i)+n \forall i \in \mathbb{Z} \quad \text { and } \quad w(1)+w(2)+\cdots+w(n)=\left(\begin{array}{c}
n+1 \\
2
\end{array}\right)
$$

For example, given $i, j \in \mathbb{Z}$ such that $i \not \equiv j$ (all congruences should be taken modulo $n$ throughout the paper), the affine transposition $t_{i, j} \in \widetilde{\mathcal{S}}_{n}$ is the periodic bijection such that $t_{i, j}(i+p \cdot n)=j+p \cdot n, t_{i, j}(j+p \cdot n)=i+p \cdot n$, and $t_{i, j}(k)=k$ for all $k \not \equiv i$ and $k \not \equiv j$ and all $p \in \mathbb{Z}$. $\widetilde{\mathcal{S}}_{n}$ is known as the affine symmetric group. It is the affine Weyl group of type $A_{n-1}$. As a Coxeter group, $\widetilde{\mathcal{S}}_{n}$ is generated by the adjacent transpositions $s_{i}=t_{i, i+1}$ for $0 \leq i<n$. If $w=s_{i_{1}} s_{i_{2}} \cdots s_{i_{p}} \in \widetilde{\mathcal{S}}_{n}$ and $p$ is minimal among all such expressions for $w$, then $s_{i_{1}} s_{i_{2}} \cdots s_{i_{p}}$ is a reduced expression for $w$ and the length of $w$ is $p$, denoted $\ell(w)=p$. The length function is the rank function for the Bruhat order on $\widetilde{\mathcal{S}}_{n}$. As a partial order, Bruhat order can be described as the transitive closure of the relation $w<t_{i, j} w$ if $\ell(w)<\ell\left(t_{i, j} w\right)$. The symmetric group $\mathcal{S}_{n}$ can be viewed as the parabolic subgroup of $\widetilde{\mathcal{S}}_{n}$ generated by $s_{1}, \ldots, s_{n-1}$.

Let $Q_{n}$ be the minimal length coset representatives for the quotient $\widetilde{\mathcal{S}}_{n} / \mathcal{S}_{n}$. Bruhat order restricted to $Q_{n}$ is again a partial order ranked by the length function. There is a rank preserving bijection from $n$-core partitions to $Q_{n}$ which respects the Bruhat order. This correspondence leads to useful criteria for Bruhat order on $Q_{n}$ in Theorem 2.3 and the covering relation in Proposition 5.3 and Corollary 5.4. We follow [29] for terminology on partial orders.

Definition 2.1. $[14,21,28]$ Define the function

$$
\mathcal{C}: Q_{n} \longrightarrow n \text {-core partitions }
$$

recursively as follows. Associate the empty partition with the identity in $Q_{n}$; namely, $\mathcal{C}(\mathrm{id})=\emptyset$. If $\mathcal{C}(w)=\lambda$ and $\ell\left(s_{i} w\right)>\ell(w)$, then $\mathcal{C}\left(s_{i} w\right)$ is obtained from $\lambda$ by adding every addable cell with residue $i$ to $\lambda$. 
In $[14,21], \mathcal{C}$ is shown to be a bijection. Denote $\mathcal{C}^{-1}$ by

$$
\mathcal{A}: n \text {-core partitions } \longrightarrow Q_{n} \text {. }
$$

Remark 2.2. Definition 2.1 can be used as an algorithm for generating $n$ core partitions. The reader is encouraged to look ahead to Figure 1 to see how the 3 -core partitions up to rank 4 are generated.

Note that if $\lambda$ is an $n$-core with an addable cell of residue $i$, then $\lambda$ has no removable cells of residue $i$. Similarly, if $\lambda$ is an $n$-core with a removable cell of residue $i$, then $\lambda$ has no addable cells of residue $i[21, \S 5]$.

The following beautiful theorem of Lascoux shows the power of the $n$ core model for $Q_{n}$.

Theorem 2.3. [23] Given $v, w \in Q_{n}$, let $\mu=\mathcal{C}(v)$ and $\lambda=\mathcal{C}(w)$ be the corresponding $n$-core partitions. Then $\mu \subset \lambda$ in Young's lattice if and only if $v<w$ in Bruhat order restricted to $Q_{n}$.

\subsection{Symmetric and quasisymmetric functions}

We adopt notations for the standard bases for $\Lambda$, the ring of symmetric functions, from [27]. For this paper, we are primarily interested in the Schur functions $s_{\lambda}$, indexed by partitions. The Schur functions form an orthonormal basis for $\Lambda$ with the Hall scalar product. The Schur functions also give the irreducible characters for representations of the general linear group as well as the Schubert basis for the cohomology of the Grassmannian [6].

We will use the expansion for Schur functions in terms of Gessel's fundamental quasisymmetric functions [8] rather than in terms of monomials on an alphabet $X=\left\{x_{1}, x_{2}, \ldots\right\}$. The $k$-Schur functions will have a similar expansion, presented in Section 3.3.

Definition 2.4. For $\sigma \in\{ \pm 1\}^{m-1}$, the fundamental quasisymmetric function associated to $\sigma$, denoted $Q_{\sigma}$, is given by

$$
Q_{\sigma}(X)=\sum_{\substack{i_{1} \leq \cdots \leq i_{m} \\ i_{j}=i_{j+1} \Rightarrow \sigma_{j}=+1}} x_{i_{1}} \cdots x_{i_{m}} .
$$

To connect quasisymmetric functions with Schur functions, for $T$ a standard tableau on $1, \ldots, m$, define the descent signature $\sigma(T) \in\{ \pm 1\}^{m-1}$ by

$$
\sigma_{i}(T)=\left\{\begin{array}{ll}
+1 & \text { if the content of } i \text { is less than the content of } i+1 \\
-1 & \text { if the content of } i+1 \text { is less than the content of } i
\end{array}\right\}
$$


Note that in a standard tableau, consecutive entries may never appear along the same diagonal so the content of the cells containing $i$ and $i+1$ are never equal. In particular, $\sigma$ is well-defined on SYT.

Theorem 2.5. [8] The Schur function $s_{\lambda}$ can be expressed in terms of quasisymmetric functions by

$$
s_{\lambda}(X)=\sum_{T \in \operatorname{SYT}(\lambda)} Q_{\sigma(T)}(X) .
$$

By Theorem 2.3, working with quasisymmetric functions instead of monomials affords us the benefit of working with standard objects instead of semistandard objects. Furthermore, the expansion in (2.6) is independent of the size of the alphabet $X$ which could be finite or infinite.

\section{3. $k$-Schur functions}

In this section, we recall two analogs of standard Young tableaux for the $n$-core poset called strong tableaux and starred strong tableaux from [17]. The spin statistic is defined on starred strong tableaux. These ingredients are combined to give the definition of $k$-Schur functions in terms of their expansion into fundamental quasisymmetric functions.

\subsection{Strong tableaux}

Consider the poset on $n$-core partitions induced from Young's lattice. A strong tableau of shape $\lambda$ is a saturated chain

$$
\emptyset \subset \lambda^{(1)} \subset \lambda^{(2)} \subset \cdots \subset \lambda^{(m)}=\lambda
$$

in the $n$-core poset from the empty tableau to $\lambda$. We denote this chain by the filling $S$ of $\lambda$ where all cells of $\lambda^{(i)} / \lambda^{(i-1)}$ contain the letter $i$.

For example, from Figure 1, the strong tableaux for $n=3$ of size $m=4$ are

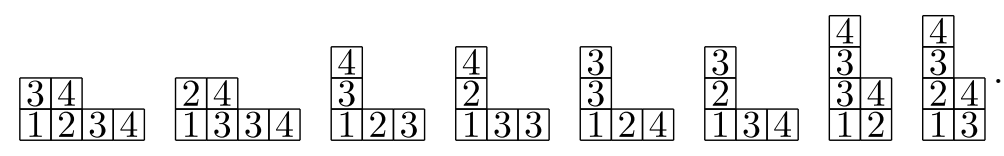




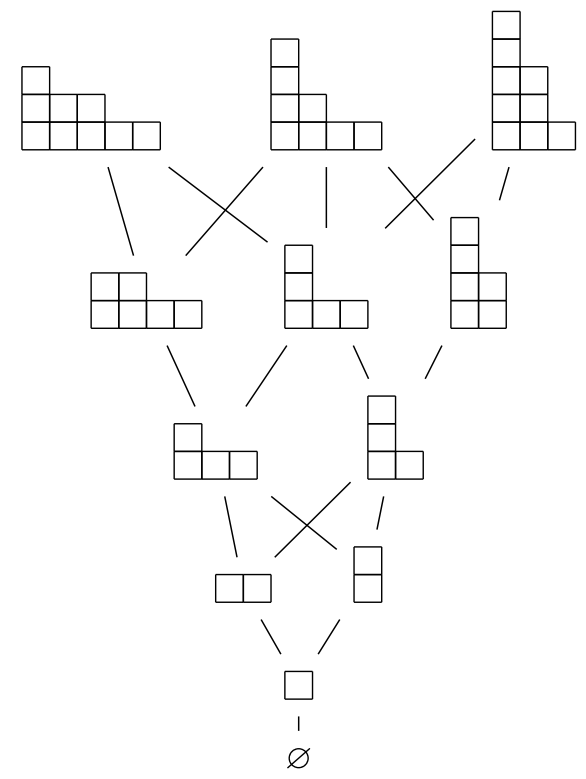

Figure 1: Poset of 3-cores up to rank 5.

\subsection{Starred strong tableaux}

A starred strong tableau, $S^{*}$, is a strong tableau $S$ where one connected component of the cells containing $i$ is chosen for each $i$, and the southeasternmost cell of the chosen components are adorned with a $*$. Therefore, the information contained in $S^{*}$ is equivalent to the pair $\left(S, c^{*}\right)$ where $c^{*}=\left(c_{1}, c_{2}, \ldots, c_{m}\right)$ is the content vector, namely $c_{i}$ is the content of the cell containing $i^{*}$.

Let $\operatorname{SST}^{*}(\lambda, n)$ be the set of all starred strong tableaux of shape $\lambda$ regarded as an $n$-core. For example, the 6 starred strong tableaux of shape $\lambda=(2,2,1,1)$ are

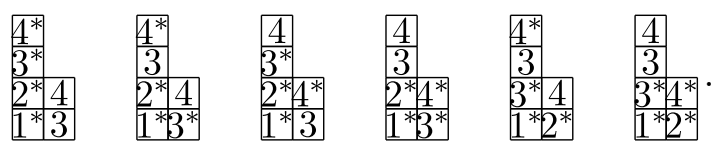

The following statistics on a starred strong tableau $S^{*}$ were first introduced in [17]. Let $n(i)$ denote the number of connected components of the cells containing $i$ of the underlying tableau $S$. Among such connected components, let $h(i)$ be the height, i.e. number of rows, of the starred connected 
component. Finally, let $d\left(i^{*}\right)$ denote the depth of $i^{*}$ in $S^{*}$, defined to be the number of components northwest of the component containing $i^{*}$. Define the statistic spin on starred strong tableaux as follows,

$$
\operatorname{spin}\left(S^{*}\right)=\sum_{i} n(i) \cdot(h(i)-1)+d\left(i^{*}\right) .
$$

For example, the spins of the starred strong tableaux in equation (3.1), from left to right, are $0,1,1,2,1,2$.

This spin statistic was dubbed "spin" based on similarities with the spin statistic on ribbon tableaux that gives LLT polynomials [24]. We explore deeper connections between LLT polynomials and $k$-Schur functions in Section 8 .

\subsection{Quasisymmetric expansion}

The $k$-Schur function $s_{\lambda}^{(k)}(X ; t)$ is the weighted generating function of starred strong tableaux of shape $\rho(\lambda)$, where $\rho$ is the bijection between $k$-bounded partitions and $k+1$-cores introduced in [22]. In was also shown that the rank of $\rho(\lambda)$ in the $n$-core poset equals $|\lambda|$ and it is conjectured that the leading term of $s_{\lambda}^{(k)}(X ; t)$ in the Schur function expansion is $s_{\lambda}(X)$.

To define $\rho$ on a $k$-bounded partition $\lambda$, from north to south slide each row of $\lambda$ east as far as necessary so that no cell has hook length greater than $k$. Filling in the resulting skew diagram gives $\rho(\lambda)$. To go back, remove all cells of $\rho(\lambda)$ with hook length greater than $k$ and re-align the rows with the western boundary. For example, we compute $\rho(3,3,2,1,1)=(5,4,2,1,1)$ when $k=4$ as follows.

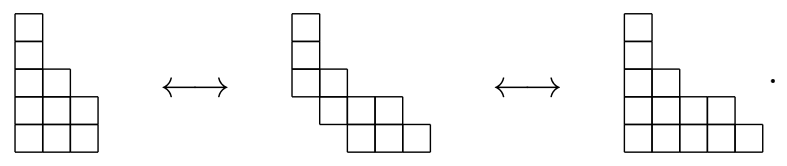

Throughout this paper, we fix $n=k+1$ so that we relate $n$-cores with $k$-Schur functions.

Rather than defining a semi-standard analog of strong tableaux to define the expansion in terms of monomials as was given in [17], we formulate the definition in terms of (standard) starred strong tableaux using quasisymmetric functions. The two versions of the definition are easily seen to be equivalent. We begin by defining the descent signature, $\sigma \in\{ \pm 1\}^{m-1}$, of a 
starred strong tableau $S^{*}$ of rank $m$ as follows.

$$
\sigma_{i}\left(S^{*}\right)= \begin{cases}+1 & \text { if the content of } i^{*} \text { is less than the content of }(i+1)^{*} \\ -1 & \text { if the content of } i^{*} \text { is greater than the content of }(i+1)^{*}\end{cases}
$$

Remark 3.1. Since the union of cells containing $i$ and those containing $i+1$ must be a valid skew shape, the southeasternmost cells containing $i$ and $i+1$ may not lie on the same diagonal. Therefore $\sigma$ is well-defined for all starred strong tableaux.

Definition 3.2. Let $\nu$ be a $k$-bounded partition. The $k$-Schur function indexed by $\nu$ is given by

$$
s_{\nu}^{(k)}(X ; t)=\sum_{S^{*} \in \operatorname{SST}^{*}(\rho(\nu), n)} t^{\operatorname{spin}\left(S^{*}\right)} Q_{\sigma\left(S^{*}\right)}(X),
$$

where the sum is over all standard starred strong tableaux of shape $\rho(\nu)$ in the $n=k+1$-core poset.

Remark 3.3. We may extend Definition 3.2 to skew strong tableaux in the obvious way by considering all saturated chains from an $n$-core $\mu$ to an $n$ core $\nu$. The definitions for starred strong tableaux and spin extend trivially to this setting. Consequently, all of our results for $k$-Schur functions also extend to this skew setting.

\section{Dual equivalence}

The main idea behind a dual equivalence graph, introduced in [2], is to provide a structure whereby the quasisymmetric functions contributing to a single Schur function are grouped together into equivalence classes, thereby demonstrating the Schur positivity of the given quasisymmetric expansion. For standard Young tableaux, the desired classes are precisely the dual equivalence classes defined by Haiman [12]. An abstract dual equivalence graph is defined by modeling the internal structure of these classes using Haiman's elementary dual equivalence relations. The connected components of a dual equivalence graph are exactly the desired equivalence classes, namely the sum over the quasisymmetric functions in a given connected component is equal to a single Schur function. Dual equivalence graphs, and more generally D graphs, provide a structure whereby we may extend the notion of dual equivalence to more general objects, in our case, starred strong tableaux. 


\subsection{Dual equivalence on standard Young tableaux}

We begin by constructing a graph on standard tableaux using dual equivalence. Originally, Haiman defined an elementary dual equivalence on three consecutive letters $i-1, i, i+1$ of a permutation by switching the outer two letters whenever the middle letter is not $i$ :

$$
\cdots i \cdots i \pm 1 \cdots i \mp 1 \cdots \cong \cdots i \mp 1 \cdots i \pm 1 \cdots i \cdots
$$

In Equation (4.1), $i \pm 1$ acts as a witness to the $i, i \mp 1$ exchange ensuring they are not adjacent letters in the permutation.

The definition of dual equivalence extends naturally to standard Young tableaux by applying the action to the permutation obtained by reading the entries along content lines. For example, the content reading word of the standard tableaux in (2.1) are 62153847 and 72153846 . These two words are dual equivalent both the 5 and the 8 act as a witness to the 6,7 exchange. Thus, the two tableaux in (2.1) are dual equivalent. Note that in a standard tableau, $i$ and $j$ may lie on the same content line only if $|i-j| \geq 3$. In particular, each of $i-1, i$ and $i+1$ must lie on distinct content lines, making equation (4.1) well-defined on standard tableaux.

It will also be helpful to think of dual equivalence on standard tableaux in terms of Young's lattice. Recall, that a standard tableau is equivalent to a saturated chain in Young's lattice with the empty partition as its unique minimal element. The rank of the empty partition is 0. If two standard tableaux $S$ and $T$ are dual equivalent via an elementary dual equivalence, then the two corresponding saturated chains differ in exactly one element, say at rank $i$. In this case, the elementary dual equivalence move interchanges $i, i+1$ and the restriction of the two chains to ranks $i-1, i, i+1$ form a subposet of Young's lattice isomorphic to $B_{2}$, the Boolean poset on subsets of $\{1,2\}$ ordered by containment. Indeed, any length two interval in Young's lattice is either isomorphic to $B_{2}$ or a chain. In the first case, there is a dual equivalence move available, and in the second case there is not.

We say that two standard tableaux are dual equivalent if one can be obtained from the other by a sequence of elementary dual equivalences. The following theorem of Haiman [12] together with Theorem 2.3 show that the sum over the quasisymmetric functions in a dual equivalence class of standard tableaux is precisely a Schur function.

Theorem 4.1. [12] Two standard tableaux of partition shape are dual equivalent if and only if they have the same shape. 


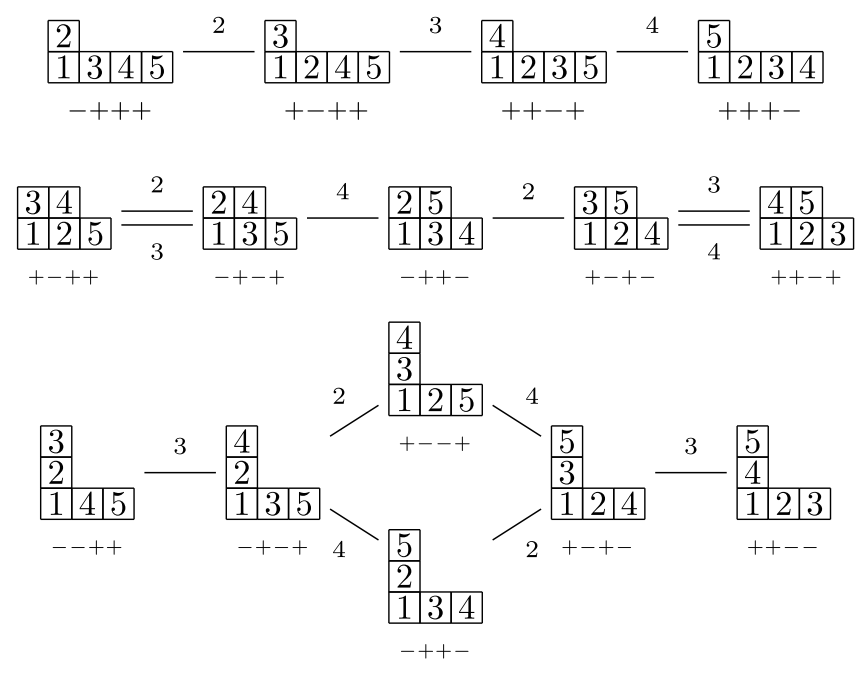

Figure 2: The standard dual equivalence graphs $\mathcal{G}_{(4,1)}, \mathcal{G}_{(3,2)}$ and $\mathcal{G}_{(3,1,1)}$.

Enrich the structure of these equivalence classes by tracking the sequence of elementary dual equivalences taking one tableau to another. Whenever $T$ and $U$ differ by an elementary dual equivalence for $i-1, i, i+1$, connect $T$ and $U$ with an edge colored by $i$. Additionally, we track the quasisymmetric function corresponding to the given tableau by writing the descent signature $\sigma(T)$, defined in Equation (2.5), below each tableaux. Let $\mathcal{G}_{\lambda}$ denote the graph on all standard tableaux of shape $\lambda$. See Figure 2 for examples of $\mathcal{G}_{\lambda}$.

Define the generating function associated to $\mathcal{G}_{\lambda}$ by

$$
\sum_{v \in V\left(\mathcal{G}_{\lambda}\right)} Q_{\sigma(v)}(X)=s_{\lambda}(X)
$$

In particular, the generating function of any vertex-signed graph whose connected components are all isomorphic to some $\mathcal{G}_{\lambda}$ is automatically Schur positive.

\subsection{Dual equivalence graphs and $D$ graphs}

Given any collection of objects with an associated signature function, the goal is to build a graph on the given objects that mimics the structure of these $\mathcal{G}_{\lambda}$. To facilitate this, we recall the local characterization of dual equivalence graphs presented in [2]. First, we need a bit of terminology. 
A signed, colored graph of degree $m$ consists of the following data: a vertex set $V$; a signature function $\sigma: V \rightarrow\{ \pm 1\}^{m-1}$; and for each $1<i<m$, a collection $E_{i}$ of unordered pairs of vertices of $V$ that represents the edges colored $i$. We denote such a graph by $\mathcal{G}=\left(V, \sigma, E_{2} \cup \cdots \cup E_{m-1}\right)$ or simply $(V, \sigma, E)$.

We say that two signed, colored graphs are isomorphic if there is a bijection between vertex sets that respects signatures and color-adjacency. Definition 4.2 gives criteria for when a signed, colored graph is isomorphic to $\mathcal{G}_{\lambda}$ by Theorem 4.3 .

Definition 4.2. A signed, colored graph $\mathcal{G}=(V, \sigma, E)$ of degree $m$ is a dual equivalence graph if the following hold:

(ax1) For $w \in V$ and $1<i<m, \sigma(w)_{i-1}=-\sigma(w)_{i}$ if and only if there exists $x \in V$ such that $\{w, x\} \in E_{i}$. Moreover, $x$ is unique when it exists.

(ax2) Whenever $\{w, x\} \in E_{i}, \sigma(w)_{i}=-\sigma(x)_{i}$ and

$$
\sigma(w)_{h}=\sigma(x)_{h} \text { if } h<i-2 \text { or } h>i+1 .
$$

(ax3) For $\{w, x\} \in E_{i}$, if $\sigma(w)_{i-2}=-\sigma(x)_{i-2}$, then $\sigma(w)_{i-2}=-\sigma(w)_{i-1}$;

$$
\text { if } \sigma(w)_{i+1}=-\sigma(x)_{i+1} \text {, then } \sigma(w)_{i+1}=-\sigma(w)_{i} \text {. }
$$

(ax4) For all $3<i<m$, every connected component of ( $V, \sigma, E_{i-2} \cup E_{i-1} \cup$ $\left.E_{i}\right)$ is either an isolated vertex or it is isomorphic to a graph in Figure 3 after the signature function is restricted to positions $[i-2, i+1]$. If $m=4$, every connected component of $\left(V, \sigma, E_{2} \cup E_{3}\right)$ is either an isolated vertex or it is isomorphic to a connected component in an induced subgraph of a graph in Figure 3 using only 2-edges and 3-edges and restricting the signature function to positions $[i-1, i+1]$.

(ax5) Whenever $|i-j| \geq 3,\{w, x\} \in E_{i}$ and $\{x, y\} \in E_{j}$, there exists $v \in V$ such that $\{w, v\} \in E_{j}$ and $\{v, y\} \in E_{i}$.

(ax6) Between any two vertices of a connected component of $\left(V, \sigma, E_{2} \cup \cdots \cup\right.$ $\left.E_{i}\right)$, there exists a path containing at most one edge in $E_{i}$.

Comparing Figure 2 with Figure 3, the largest possible connected components of $\left(V, \sigma, E_{i-2} \cup E_{i-1} \cup E_{i}\right)$ are exactly the graphs for $\mathcal{G}_{\lambda}$ when $\lambda$ is a partition of 5 . Taking this comparison to its ultimate conclusion yields the following result. 


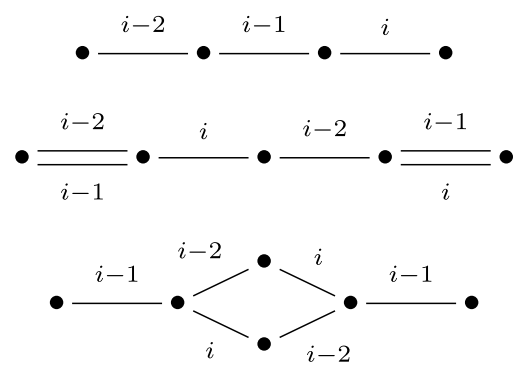

Figure 3: Possible 3-color connected components of a dual equivalence graph with at least two vertices. Isolated vertices are also possible.

Theorem 4.3. [2] For $\lambda$ a partition of $m, \mathcal{G}_{\lambda}$ is a dual equivalence graph of degree $m$. Moreover, every connected component of a dual equivalence graph of degree $m$ is isomorphic to $\mathcal{G}_{\lambda}$ for a unique partition $\lambda$ of $m$.

In practice, Axioms 1,2 and 5 are trivially verified if $E_{i}$ is the set of pairs $\left\{\left(w, \phi_{i}(w)\right): w \neq \phi_{i}(w)\right\}$ determined by a family of involutions $\phi_{i}: V \longrightarrow V$ such that for all $w \in V$ :

1. If $\sigma(w)_{i-1, i}=+-$, then $\sigma\left(\phi_{i}(w)\right)_{i-1, i}=-+$, and vice versa.

2. Fixed points of $\phi_{i}$ are precisely those $w$ such that $\sigma(w)_{i-1, i}=++$ or -- .

3. The signatures $\sigma(w)$ and $\sigma\left(\phi_{i}(w)\right)$ agree outside the range of indices $i-2 \leq j \leq i+2$.

4. The involutions $\phi_{i}$ and $\phi_{j}$ commute whenever $|j-i| \geq 3$.

Axiom 3 is typically verified by keeping track of a witness in each case. The real difficulty lies in Axioms 4 and 6.

In [2], the first author extended the notion of dual equivalence in order to apply it to the LLT and Macdonald polynomials. For the extension, Axiom 6 is no longer required and Axiom 4 is replaced by a weaker axiom. Using this generalized notion of dual equivalence, it was shown that connected components have Schur positive generating functions but not necessarily a single Schur function. We use the same technique here to prove that the terms in a $k$-Schur function can be partitioned in connected components of a graph which are locally Schur positive. Therefore, we review the necessary material from [2] below.

Definition 4.4. Let $\mathcal{G}=(V, \sigma, E)$ be a signed, colored graph. Define the generating function associated to $\mathcal{G}$ to be 


$$
F_{\mathcal{G}}(X)=\sum_{v \in V} Q_{\sigma(v)}(X)
$$

Definition 4.5. A signed, colored graph $\mathcal{G}=(V, \sigma, E)$ of degree $m$ is a $D$ graph if Axioms 1, 2, 3 and 5 (from Definition 4.2) hold for $\mathcal{G}$. A D graph is said to be locally Schur positive on $h$-colored edges, denoted $\mathrm{LSP}_{h}$, provided for all $2 \leq h<i<m$ :

$\left(\mathrm{LSP}_{h}\right)$ Every connected component of $\left(V, \sigma, E_{i-h+1} \cup \cdots \cup E_{i}\right)$ using $h$ consecutive edge sets with signatures restricted to positions $[i-h, i+1]$ has a symmetric and Schur positive generating function.

For example, all of the graphs on Page 396 are locally Schur positive on 2-colored edges and 3-colored edges. Notice that any D graph satisfying Axioms 4 and 6 necessarily implies the graph is $\mathrm{LSP}_{h}$ for all $h$ by Theorem 4.3.

Observe that the signature function of a $\mathrm{D}$ graph can be recovered from the edges plus a single sign in any one signature on any one vertex via the axioms. Thus each graph in Figure 3 can be assigned signature functions in exactly 2 ways which make them into a D graph. The third graph can only be signed in one way up to isomorphism.

\section{Poset on $n$-cores}

In order to define an analog of dual equivalence for starred strong tableaux, we must first understand saturated chains in the $n$-core poset. In this section, we do this by exploiting the connection between $n$-cores and $\widetilde{\mathcal{S}}_{n}$ using the abacus model for partitions.

\subsection{Covering relations}

We can describe the $n$-core poset more directly using the abacus model for cores from [14]. Consider the diagram of a partition $\lambda$, not necessarily an $n$-core, lying in the $\mathbb{N} \times \mathbb{N}$ plane with infinite positive axes. Walk in unit steps along the boundary of $\lambda$ placing a bead $(\bullet)$ on each vertical step and a spacer (o) on each horizontal step. Then straighten the boundary to get a doubly infinite rod with the main diagonal marked by a vertical line. This gives the binary string uniquely representing $\lambda$ when beads are replaced by 1 's and spacers by 0's. For example, we construct the string for $(4,2)$ as follows. 


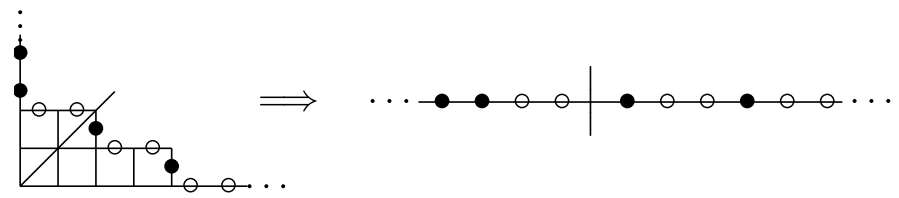

Define the content of a bead or spacer to be the content of the diagonal immediately southeast. Indexing each bead or spacer by its content gives an injective map from partitions to binary strings. The abacus associated to $\lambda$ is the binary string of $\lambda$ with beads and spacers indexed by their content.

Remark 5.1. Given any doubly infinite binary string $s$ such that $s_{i}$ is a bead for all $i<l$ and $s_{i}$ is a spacer for all $i>r$ for some $l, r$, there is a unique re-indexing of $s$ making it an abacus associated to a (unique) partition.

Interchanging a bead on the abacus of $\mu$ with a spacer $m$ places to its right corresponds to adding a ribbon of length $m$ to $\mu$, and similarly interchanging a bead with a spacer $m$ positions to its left removes an $m$ ribbon from $\mu$. In particular, if the moving bead lands in position $s$, then the head of the added ribbon will have content $s-1$.

Divide the abacus into $n$ rods, each containing all beads and spacers of the same residue. Removing an $n$-ribbon from the boundary of $\lambda$ precisely corresponds to moving a bead left along its rod. Therefore $\lambda$ is an $n$-core precisely when each rod is an infinite string of beads followed by an infinite string of spacers. Define the content of a rod to be the content of the bead or spacer immediately to the right of the vertical line marking the main diagonal. We will identify a rod by its content throughout the paper. Continuing with the previous example, taking $n=3$ gives the following abacus decomposition of $(4,2)$, showing rods 1,2 and 3 .
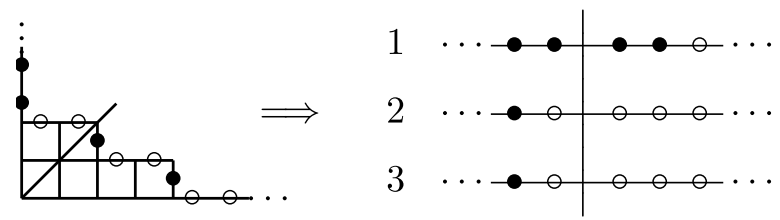

Remark 5.2. Rotating the bottom row of the $n$-rod abacus for $\mu$ to the top and shifting all beads in that row one column to the right will again represent the abacus for $\mu$, but now shifted so that the rods have contents $0, \ldots, n-1$ from top to bottom. Similarly, rotating the top row down to the bottom and shifting all beads left on that row gives the $n$-rod abacus for $\mu$ with contents $2, \ldots, n+1$. Thus, the abacus can be represented by $n$ rods 
of contents $k, k+1, k+2, \ldots, k+n-1$ for any integer $k$ by scrolling up or down.

Define the length of each rod of the $n$-rod abacus as follows. For $i=$ $1,2, \ldots, n$, define the length of the rod with content $i$ to be the number of beads on the rod with positive content minus the number of spacers on the rod with nonpositive content (at most one of these numbers is nonzero). For example, the lengths of rods $1,2,3$ for the 3 -core $(4,2)$ are $2,-1,-1$. In line with Remark 5.2, define the length of the remaining rods by setting the length of $\operatorname{rod} i-n$ equal to one plus the length of $\operatorname{rod} i$. It is sometimes convenient to rescale the lengths of the rods so that the rods $1,2, \ldots, n$ have nonnegative length with at least one having length 0 . For now, we are concerned only with the relative lengths of the rods.

Affine permutations act on $n$-core partitions as discussed in Section 2.2. This action can be stated in terms of abaci as well. Recall we can represent a partition by an infinite binary string. Since affine permutations are bijections from $\mathbb{Z}$ to $\mathbb{Z}$, we can apply such a bijection to any binary string. If the binary string represents an $n$-core then any affine transposition applied to the binary string will also represent an $n$-core. We leave it to the reader to verify this action is consistent with the action of simple affine transpositions acting on $n$-cores described earlier. In particular, the action of an affine transposition on an $n$-core can be thought of pictorially as exchanging two rods of its abacus and modifying all $n$-translates of these two rods accordingly. The following observations, also noted in [17], follow easily from the abacus model.

Proposition 5.3. The following statements hold for an $n$-core $\mu$ and $t_{r, s} \in$ $\widetilde{\mathcal{S}}_{n}$ with $r<s, r \not \equiv s$ :

1. The abacus for $t_{r, s} \mu$ is obtained from the abacus for $\mu$ by swapping the lengths of the two rods with contents $r$ and $s$. All rods with content distinct from $r, s \bmod n$ have the same length in $\mu$ and $t_{r, s} \mu$.

2. In the $n$-core poset, $t_{r, s} \mu>\mu$ if and only if the rod of content $r$ has larger length than the rod of content $s$ in $\mu$.

3. An $n$-core $\lambda$ covers $\mu$ if and only if $\lambda=t_{p, q} \mu$ for some pair $p<q, p \not \equiv q$ such that in the abacus for $\mu$ there is a bead at position $p$, a spacer at position $q$, and no rod between $p$ and $q$ has length weakly between the length of rod $p$ and the length of rod $q$. Furthermore, the head and tail of one ribbon in $\lambda / \mu$ have contents $q-1$ and $p$ respectively.

Proof. The first statement follows form the action of an affine permutation on infinite binary strings. The second statement is immediate since moving 
beads right adds ribbons and moving beads left removes ribbons. The third statement also follows from this interpretation.

Proposition 5.3 is enough to describe precisely what $\lambda / \mu$ may look like when $\lambda$ covers $\mu$ in the $n$-core poset. The condition on the lengths of the rods that lie between the interchanging rods of the abacus implies that the connected components of $\lambda / \mu$ are identical ribbons. By Remark 5.2, the two rods being exchanged must have distinct residues and no rod between them may have the same residue as either of them. The contents across which the beads move determine the contents contained in the ribbons, and the fact that both rods are beads followed by spacers ensures that the ribbons lie on consecutive residues. These observations reprove the following statement due to Lam, Lapointe, Morse and Shimozono.

Corollary 5.4. [17, Prop. 9.5] Let $\mu$ be an n-core and $t_{r, s}$ an affine transposition such that $t_{r, s} \mu$ covers $\mu$ in the $n$-core poset. Then $0<s-r<n$ and the connected components of $t_{r, s} \mu / \mu$ are identical shape ribbons with cell residues from $r \bmod n$ to $s-1 \bmod n$. Moreover, if rod $r$ has $k>0$ more beads than rod $s$, then $t_{r, s} \mu / \mu$ has exactly $k$ identical ribbons. If the head of the first ribbon lies in a cell with content $c$, then the head of the other ribbons have content $c+n, c+2 n, \ldots, c+(k-1) n$.

By Corollary 5.4, for a strong tableau $S$ of shape $\lambda$, call the connected components of $\lambda_{i} / \lambda_{i-1}$ the $i$-ribbons of $S$. Recall from Section 3.2 that a starred strong tableau consists of a strong tableau plus a choice of $i$-ribbon for each $i$ present in $S$. We use the next definition and corollary to relate the starred strong tableaux to saturated chains labeled by certain sequences of transpositions.

Definition 5.5. Let $\mu \subset \lambda$ be $n$-cores, and let $T(\lambda / \mu, n)$ be the set of all transposition sequences $\left(t_{r_{1} s_{1}} \rightarrow t_{r_{2} s_{2}} \rightarrow \cdots \rightarrow t_{r_{m} s_{m}}\right)$ such that

1. the product $t_{r_{m} s_{m}} \cdots t_{r_{2} s_{2}} t_{r_{1} s_{1}} \mu=\lambda$ as elements of $\widetilde{\mathcal{S}}_{n} / \mathcal{S}_{n}$;

2. for each $1 \leq i \leq m$, we have $0<s_{i}-r_{i}<n$;

3. for each $0 \leq i<m$, the abacus for $\mu^{(i)}=t_{r_{i} s_{i}} \cdots t_{r_{2} s_{2}} t_{r_{1} s_{1}} \mu$ contains a bead at position $r_{i+1}$, a spacer at position $s_{i+1}$, and every rod with content between $r_{i+1}$ and $s_{i+1}$ has length strictly smaller than both the length of rod $r_{i+1}$ and the length of rod $s_{i+1}$ or strictly larger than both.

By Proposition 5.3, condition (3) above implies $\mu=\mu^{(0)}<\mu^{(1)}<\cdots<$ $\mu^{(m)}=\lambda$ forms a saturated chain in the $n$-core poset. The following is a consequence of Proposition 5.3 and Corollary 5.4. 
Corollary 5.6. Let $\mu \subset \lambda$ be n-cores. There exists a bijection from skew starred strong tableaux $S^{*} \in \operatorname{SST}^{*}(\lambda / \mu, n)$ to $T(\lambda / \mu, n)$ given by mapping

$$
S^{*} \mapsto\left(t_{r_{1} s_{1}} \rightarrow t_{r_{2} s_{2}} \rightarrow \cdots \rightarrow t_{r_{m} s_{m}}\right)
$$

where $s_{i}-1$ and $r_{i}$ are the contents of the head and tail of the $i$-ribbon containing $i^{*}$ in $S^{*}$.

For example, this bijection maps

$$
\underset{\frac{1}{3}}{1^{*} 2^{*} *^{*}} \mapsto\left(t_{0,1} \rightarrow t_{1,2} \rightarrow t_{2,3} \rightarrow t_{-2,-1}\right) .
$$

\subsection{Intervals of length two}

As motivation, recall that an elementary dual equivalence on standard tableaux may be defined in terms of interval exchanges in Young's lattice. Though the induced poset on $n$-cores in not as nice as Young's lattice, Björner and Brenti [5] showed that any interval of length two is either a chain or isomorphic to $B_{2}$.

Definition 5.7. Let $S=\left(\emptyset=\mu^{0} \subset \mu^{1} \subset \cdots \subset \mu^{m}\right)$ be a saturated chain in the $n$-core poset such that the interval $\left[\mu^{i-1}, \mu^{i+1}\right]$ is not a chain for $0<i<m$. The $i$-interval swap on $S$, denoted $\operatorname{swap}_{i, i+1}(S)=\operatorname{swap}_{i+1, i}(S)$, replaces $\mu^{i}$ with the unique other $n$-core at rank $i$ in $\left[\mu^{i-1}, \mu^{i+1}\right]$.

For example, from Figure 1 we see that a 2-interval swap on the chain

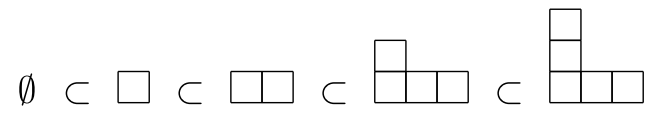

results in the chain

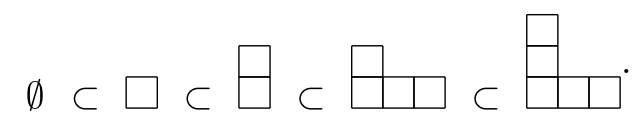

In terms of the strong tableaux, the same 2-interval swap gives

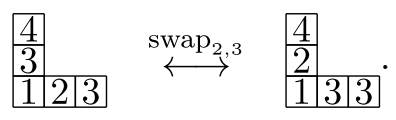




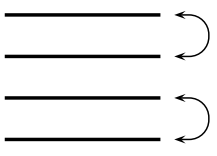

disjoint

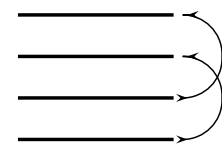

interleaving

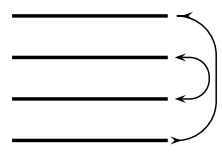

nested

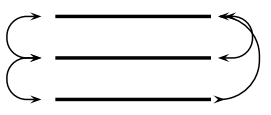

abutting

Figure 4: Possible rod exchanges for a length two interval.

By Definition 5.5, the same two saturated chains can be represented by the following transposition sequence

$$
\operatorname{swap}_{2,3}\left(t_{0,1} \rightarrow t_{1,2} \rightarrow t_{2,3} \rightarrow t_{-2,-1}\right)=t_{0,1} \rightarrow t_{-1,0} \rightarrow t_{1,3} \rightarrow t_{-2,-1},
$$

where only the two transpositions in the middle are modified. In general, the map $\operatorname{swap}_{i, i+1}(S)$ always modifies the $i$ th and the $i+1$ st transpositions in any transposition sequence representing the saturated chain $S$ and leaves all other transpositions in the sequence fixed. The two new transpositions are not unique however since we have not yet described how the stars will move. This extension will be called a bswap and introduced in Section 6. First, we need a complete understanding of how the $i$-ribbons and the $i+1$-ribbons can appear in a strong tableau and how they change under an $i$-interval swap.

Using the abacus model for cores and Proposition 5.3, we can explicitly describe the result of an $i$-interval swap on a strong tableau $S=\left(\emptyset \subset \mu^{1} \subset\right.$ $\left.\cdots \subset \mu^{m}\right)$ in terms of the two rod exchanges corresponding to the covering relations in the interval $\left[\mu^{i-1}, \mu^{i+1}\right]$. In order to analyze two consecutive rod exchanges, we extend the $n$-rod abacus picture to include extra rods above as necessary so that the four rods to be exchanged all appear as rows of the picture with the longer row above the shorter row. Ignoring the rods which are untouched by the exchange and choosing representatives of the exchanging rods as close together as possible, there are four natural cases to consider, each depicted in Figure 4: disjoint, interleaving, nested and abutting. There are three possible ways for the rod exchanges to be abutting; the two depicted and also the reverse of the right hand side. For the first three cases in Figure 4, the corresponding transpositions will have four distinct residues whereas for the abutting case, they will have only two or three distinct residues.

The easiest case to consider is a disjoint exchange. Here we assume all of the residues of the rods to be exchanged are distinct, lest we actually have an abutting exchange. Further, we can assume the exchanging rods have 
contents $a<b<c<d$ with $n>d-a>0$ since the rods are as close together as possible. The two exchanges in this case clearly commute, and taking either first raises the rank by exactly one by Corollary 5.4. In the strong tableau, such an $i$-interval swap will happen precisely when the cells of the $i$-ribbons and $(i+1)$-ribbons have no residues in common, and the effect of the swap will be to exchange all $i$ 's for $i+1$ 's and conversely.

The case of an interleaving exchange is only slightly more interesting, though the conclusion of this case is noteworthy. Labeling the residues of the exchanging rods $a<b<c<d$ from top to bottom, again we assume all four residues to be distinct lest we be pulled into the abutting case. The assumption that these two exchanges each increase the rank in the poset forces rod $a$ longer than $\operatorname{rod} c$ and similarly rod $b$ longer than rod $d$ by Proposition 5.3. Suppose $\mu^{i-1} \subset t_{a, c} \mu^{i-1}=\mu^{i} \subset t_{b, d} t_{a, c} \mu^{i-1}=\mu^{i+1}$; the other case is similarly resolved. By Proposition 5.3, this means the length of rod $b$ does not lie between the lengths of rods $a$ and $c$ and that the length of rod $a$ does not lie between the lengths of rods $b$ and $d$. Recall, we chose a picture for the abacus so that the length of rod $b$ is larger than the length of rod $d$ and the length of rod $a$ is longer than the length of rod $c$. These statements together imply that the lengths of rods $a$ and $c$ do not interleave the lengths of rods $b$ and $d$, and so the transpositions taken in the other order each raise the rank by exactly one, thus $\mu^{i-1} \subset t_{b, d} \mu^{i-1} \subset t_{a, c} t_{b, d} \mu^{i-1}=\mu^{i+1}$ is a valid strong tableau. In this new strong tableau, the contents of the $i$ ribbons and $i+1$-ribbons will not overlap, though the residues will. It is also important to note that the $i$-ribbons and $i+1$-ribbons will not have the same residues for their heads or tails. In this case, the $i$-interval swap again simply exchanges all $i$ 's for $i+1$ 's and conversely. We summarize the key observation in this case as follows.

Proposition 5.8. An $i$-ribbon and an $i+1$-ribbon in a strong tableau have overlapping contents if and only if the contents of one ribbon are strictly contained in the contents of the other. Furthermore, the contents of the head and tail of the longer ribbon do not occur among the contents of the shorter ribbon.

More generally, we say two ribbons are nested if the second condition of Proposition 5.8 holds. We also say two ribbons $R_{1}$ and $R_{2}$ are independent if $R_{1} \cup R_{2}$ has two connected components as a skew shape.

In the case of a nested exchange, again label the rod contents $a<b<$ $c<d$ from top to bottom. We can assume $d-a<n$ by Corollary 5.4. Here the two corresponding transpositions commute, and each will raise the rank by exactly one. The interesting feature of this case lies in way the nested 

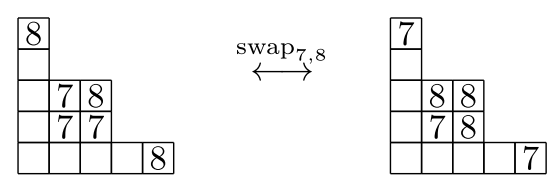

Figure 5: A nested exchange is show on two 4-core skew strong tableaux.

ribbons can appear in the corresponding strong tableaux. See Figure 5 for example.

By Proposition 5.3, neither the length of rod $b$ nor the length of $\operatorname{rod} c$ may lie between the lengths of rods $a$ and $d$. If both $\operatorname{rod} b$ and $\operatorname{rod} c$ are longer than $\operatorname{rod} a$ or both shorter than $\operatorname{rod} d$ (necessarily rod $a$ is longer than $\operatorname{rod} d$ ), then the $i$-ribbons and $i+1$-ribbons will have no contents in common, though the residues of one ribbon will be strictly contained within the residues of the other. Furthermore, both the heads and tails of the $i$ ribbons and $i+1$-ribbons have distinct residues.

On the other hand, if $\operatorname{rod} b$ is longer than $\operatorname{rod} a$ and $\operatorname{rod} c$ is shorter than $\operatorname{rod} d$ (necessarily rod $b$ is longer than $\operatorname{rod} c$ ), then the content of every instance of the longer ribbon (corresponding to $t_{a, d}$ ) overlaps the content of a shorter ribbon (corresponding to $t_{b, c}$ ) and there must be an instance of the shorter ribbon containing a cell of content $b-1$ which occurs independently from all of the longer ribbons. An $i$-interval swap changes all entries in all of the shorter ribbons that appear independently of the longer ribbons and all entries of the longer ribbons that are not on the same content as a shorter ribbon.

For example, we can apply a nested exchange to the 4-core partition $(4,1,1,1)$ encoded as an abacus with 4 rods, numbered $1,2,3,4$, with lengths $(-1,0,0,1)$. The nested exchange is more clear if we instead consider the same abacus but with rods numbered $-1,0,1,2$ by scrolling $(-1,0,0,1)$ back two steps to $(1,2,-1,0)$ via Remark 5.2 . Now, we are in the case where $a=-1, b=0, c=1, d=2, \operatorname{rod} a$ has length $1, \operatorname{rod} b$ has length $2, \operatorname{rod} c$ has length -1 , and rod $d$ has length 0 . These rod lengths satisfy the conditions of a nested exchange of the second type described above. Applying the nested exchange corresponding to the two commuting transpositions $t_{-1,2} t_{0,1}$ results in rod lengths $(0,-1,2,1)$ which encode the 4 -core partition $(5,3,3,1,1)$. In terms of strong tableaux, the two transposition sequences $t_{-1,2} \rightarrow t_{0,1}$ and $t_{0,1} \rightarrow t_{-1,2}$ applied to $\mu=(4,1,1,1)$ are related by $\operatorname{swap}_{7,8}$ as shown below. This discussion proves the following lemma. 
Lemma 5.9. If an $i$-ribbon and an $i+1$-ribbon are nested, then

1. At least two copies of the shorter ribbon occur independently from the longer ribbon, with at least one on either side of the consecutive sequence of copies of the longer ribbon.

2. Every copy of the longer ribbon nests a copy of the shorter ribbon.

3. Both the heads and tails of the $i$-ribbons and $i+1$-ribbons have distinct residues.

4. An i-interval swap is possible.

The final case of an abutting exchange will involve exactly three distinct indices on the transpositions, though possibly only two distinct residues. Let $\mu$ be an $n$-core partition represented as an $n$ rod abacus with three highlighted rods among them with contents $a<b<c$ from top to bottom on which we can apply an abutting exchange. Suppose that the three residues of $a, b, c$ are all distinct. This is necessarily the case for the right hand side of Figure 4. Say the two exchanges correspond with the transposition sequence $\left(t_{a, c} \rightarrow t_{a, b}\right)$. Then by Proposition 5.3, we know rod $a$ is strictly longer than rod $c$. Similarly, since we can apply $t_{a b}$ to the partition $t_{a c} \mu$, we also know that $\operatorname{rod} c$ is longer than $\operatorname{rod} b$, so the three rod lengths are totally ordered. We note that $t_{b, c} t_{a, b}=t_{a, b} t_{a, c}$ so this equation along with the total order on the lengths of the rods ensures that an interval swap is possible. The new transposition sequence after applying this interval swap would be $\left(t_{a, b} \rightarrow\right.$ $\left.t_{b, c}\right)$ which corresponds with the left hand side of the abutting exchange pictured in Figure 4. If the two exchanges correspond with the transposition sequence $\left(t_{a, b} \rightarrow t_{a, c}\right)$, examining the required rod length inequalities again we see that $\left(t_{b, c} \rightarrow t_{a, b}\right)$ is a valid transposition sequence on the same rank 2 interval. This again corresponds with the left hand side of the abutting exchange pictured in Figure 4. If the right hand side of Figure 4 is turned upside down, a similar analysis holds. Furthermore, the interval swaps form an involution on the two chains in any interval isomorphic to $B_{2}$ so we have covered all possible cases of an abutting exchange in the form of the left hand side of the abutting picture of Figure 4 as well. Hence in all cases of an abutting exchange with three distinct residues, there exists an interval swap determined above.

Assuming that $\left[\mu^{i-1}, \mu^{i+1}\right]$ is isomorphic to $B_{2}$, one way to recognize if an abutting exchange is required for $\operatorname{swap}_{i, i+1}(S)$ is that an $i$-ribbon and an $i+1$-ribbon together form a ribbon shape. In this case, we will say these two ribbons abut each other. From the transpositions pictured in the abutting case of Figure 4 and Corollary 5.4, we observe that the sum of the lengths of an $i$-ribbon and an $i+1$-ribbon is necessarily less than $n$ and exactly 
one of the two ribbon types occurs without abutting a copy of the other. In this instance, the $i$-interval swap will change all entries of the non-abutting ribbons and all entries in their $n$-translates. For example, the 2-ribbon abuts a 3-ribbon in the strong tableau on the left in (5.1).

The other way to recognize if an abutting exchange is required for $\operatorname{swap}_{i, i+1}(S)$ is that, among the $i, i+1$-ribbons, one ribbon is strictly longer than the other and the longer ribbon contains an $n$-translate of the shorter and the heads or tails of the two ribbons have the same residue depending on if the shared rod is $a$ or $c$. See for example, the 2-ribbon and 3-ribbon in the strong tableau on the right in (5.1). Here an interval swap will change all entries of the shorter ribbons and all entries of the longer ribbons that are not part of an $n$-translate of the shorter. This case is also recovered from the left hand side of Figure 4 when the lengths of the three rods are all distinct; we omit details as the case is completely parallel.

Following the details of the abutting exchange case carefully, we have the following.

Proposition 5.10. Suppose $\operatorname{swap}_{i, i+1}(S)$ is obtained from $S$ by an abutting exchange. Assume the corresponding transpositions are indexed by 3 distinct residues mod $n$. Then either

- No i-ribbon abuts any $i+1$-ribbon, but one of these ribbons strictly contains an n-translate of the other with a shared head or tail occurring on a consecutive residue.

- OR, all instances of one ribbon type abut the other while the other will also have at least one components which is non-abutting and the sum of the length of an $i$-ribbon and an $i+1$-ribbon is at most $n-1$. In this case, if an $i+1$-ribbon abuts an $i$-ribbon from the north, then the non-abutting ribbons lie always southeast of the abutting ribbons, and if an $i$-ribbon abuts an $i+1$-ribbon from the west, then the non-abutting ribbons lie always northwest of the abutting ribbons.

Finally, consider an abutting exchange as in the left hand side of the abutting case in Figure 4. If the three rod lengths are distinct and the three residues are distinct, then the exchange is covered by Prop 5.10. In each of the remaining cases, we claim the interval $\left[\mu^{i-1}, \mu^{i+1}\right]$ is a chain so an $i$-interval swap is not possible.

Proposition 5.11. Let $S=\left(\emptyset=\mu^{0} \subset \mu^{1} \subset \cdots \subset \mu^{m}\right)$ be a saturated chain in the $n$-core poset. Then the interval between $\mu^{i-1}$ and $\mu^{i+1}$ in the $n$-core poset is a chain if and only if each $i$-ribbon abuts an $i+1$-ribbon and each $i+1$-ribbon abuts an $i$-ribbon. Moreover, the length of an $i$-ribbon plus the 
length of a $i+1$-ribbon is less than or equal to $n$, with equality if and only if $\mu^{i+1} / \mu^{i-1}$ is a single connected ribbon shaped component starting and ending with $i+1$-ribbons.

Proof. Assume $\left[\mu^{i-1}, \mu^{i+1}\right]$ is a chain. Then by the previous analysis of two consecutive rod exchange cases examined in this section above, one can show that a length 2 chain corresponds with a transposition sequence of the form $t_{b, c} \rightarrow t_{a, b}$ with $a<b<c$ or $a>b>c$.

Assume $a$ and $c$ have different residues (both necessarily have distinct residues from $b)$. In this case, $t_{a, b} t_{b, c}=t_{a, c} t_{a, b}$, and by Proposition 5.3 we can assume $0<c-a<n$. Thus, we see from the $n$-rod abacus model that the skew shape $\mu^{i+1} / \mu^{i-1}$ is the union of a positive number of $n$-translates of a single ribbon shape of length less than $n$ and none of these ribbons overlap in content. More precisely, $i$-ribbons and $i+1$-ribbons always occur in connected pairs and the sum of their lengths is strictly less than $n$. For example, the 3-ribbons and 4-ribbons in the first strong tableau in (3.1) have this property, and interpreting this strong tableau as a saturated chain one can see the corresponding chain property from the poset in Figure 1 on ranks 2 to 4 .

If, on the other hand, $a$ and $c$ have the same residue, then we can assume $c=a+n$ by choosing to label the exchanging rods as close together as possible. Hence, the length of the ribbons corresponding to $t_{a, b}$ and those corresponding to $t_{b, a+n}$ necessarily add to $n$ so $\mu^{i+1} / \mu^{i-1}$ is a single connected ribbon shaped component. Furthermore, recall that $\operatorname{rod} c$ is one shorter than the length of $\operatorname{rod} a$ by Remark 5.2 and the fact $c=a+n$. If $\operatorname{rod} b$ is shorter than $\operatorname{rod} a$, then the chain corresponds with the transposition sequence $t_{a, b} \rightarrow t_{b, c}$, otherwise the transpositions happen in the reverse order. In either case, by considering how ribbons are created using the abacus model and Proposition 5.3, we observe that the ribbon $\mu^{i+1} / \mu^{i-1}$ is tiled by an alternating sequence of $i$-ribbons and $i+1$-ribbons and it begins and ends with an $i+1$-ribbon. The last tableau in (3.1) has this property on the 3 -ribbons and 4-ribbons, as does the strong tableau underlying both pictures in Figure 10 on the 2, 3-ribbon and the 1, 2-ribbon.

To prove the reverse direction, assume each $i$-ribbon abuts an $i+1$ ribbon and conversely. Then by Corollary 5.4 we can infer that the chain $\mu^{i-1} \subset \mu^{i} \subset \mu^{i+1}$ corresponds to an abutting exchange. If all three contents of the exchanging rods have distinct residues, then either $\left[\mu^{i-1}, \mu^{i+1}\right]$ is a chain or we would find a contradiction to the second case of Proposition 5.10.

If there are only two distinct indices among the exchanging rods then the relative lengths of these rods determine the only possible exchange sequence taking $\mu^{i-1}$ to $\mu^{i+1}$ by Proposition 5.3. Thus, $\left[\mu^{i-1}, \mu^{i+1}\right]$ is again a chain. 
Table 1: Summary of length two interval types

\begin{tabular}{|c|c|c|l|l|}
\hline \#res & \#dis & Type & Exchange & Skew Shape \\
\hline 2 & 2 & $C_{3}$ & abutting & $\begin{array}{l}\text { One long ribbon alternating } i \text { 's and } \\
i+1 \text { 's, starting and ending with } i+1 .\end{array}$ \\
\hline 3 & 2 & $C_{3}$ & abutting & $\begin{array}{l}\text { Every component is an identical ribbon } \\
\text { composed of one } i \text {-ribbon abutting one } \\
i+1 \text { ribbon. }\end{array}$ \\
\hline 3 & 3 & $B_{2}$ & abutting & $\begin{array}{l}\text { Long ribbons contain } n \text {-translate of shor- } \\
\text { ter ribbons. One short ribbon occurs } \\
\text { independently. }\end{array}$ \\
\hline 4 & $2,3,4$ & $B_{2}$ & disjoint & $\begin{array}{l}\text { All ribbons are non-overlapping, } \\
i \text {-ribbons don't abut } i+1 \text {-ribbons or vice } \\
\text { versa. No } n \text {-translate of one ribbon type } \\
\text { overlaps the other. }\end{array}$ \\
\hline 4 & 4 & $B_{2}$ & interleaving & $\begin{array}{l}\text { All ribbons are non-overlapping, } i \text {-ribbons } \\
\text { don't abut } i+1 \text {-ribbons or vice versa. } \\
\text { Some } n \text {-translate of an } i \text {-ribbon overlaps } \\
\text { an } i \text { - 1-ribbon with distinct heads and } \\
\text { tails. }\end{array}$ \\
\hline
\end{tabular}

Corollary 5.12. If a strong tableau $S=\left(\mu^{0} \subset \mu^{1} \subset \mu^{2}\right)$ is the result of an abutting exchange, then $\mu^{2} / \mu^{0}$ is the union of ribbons with nonoverlapping content. If every ribbon in $\mu^{2} / \mu^{0}$ is an identical $n$-translate of the first, then the interval $\left[\mu^{0}, \mu^{2}\right]$ is a chain.

Proof. This follows from the characterization of all abutting exchanges in this subsection, Propositions 5.10 and 5.11.

Table 1 summarizes the discussion above characterizing all possible length two intervals determined by two consecutive rod exchanges. Assume the initial $n$-core is $\mu$. First apply $t_{a, b}$ then $t_{c, d}$ assuming $0<b-a<n$, $0<d-c<n, \operatorname{rod} a$ longer than $\operatorname{rod} b, \operatorname{rod} c$ longer than $\operatorname{rod} d$, and all 4 indices appear in the smallest possible interval of $\mathbb{Z}$ which satisfies these conditions. Let \# res be the number of distinct residues among $a, b, c, d \bmod$ 
$n$. Let \#dis be the number of distinct rod lengths among rods $a, b, c, d$ in $\mu$. The interval $\left[\mu, t_{c d} t_{a b} \mu\right]$ is either isomorphic to $B_{2}$ or the chain $C_{3}$ with 3 elements. The two interval types are distinguished by considering \#res and \#dis or equivalently by considering the skew shape as partitions of $t_{c d} t_{a b} \mu / \mu$.

\section{Affine dual equivalence}

We now have all the ingredients to construct an analog of dual equivalence for starred strong tableaux, which we call affine dual equivalence. Though our equivalence relation will not share all of the properties of dual equivalence on tableaux, we will go on in Section 7 to construct a signed colored graph from our elementary equivalence relations that we show to be a D graph.

While the elementary equivalence relations will have a somewhat complicated description, there are essentially only two cases: one that precisely mirrors dual equivalence, and another that is a close approximation when the former is not applicable. Remarkably, the relations also preserve the spin statistic on starred strong tableaux.

\subsection{Elementary equivalences}

In this subsection, we describe a family of involutions $\varphi_{i}$ on all starred strong tableaux of a given shape that will define the elementary affine dual equivalence on $i-1, i, i+1$. Recall that a starred strong tableau $S^{*}$ of shape $\lambda$ can be represented by a strong tableau $S=\left(\emptyset \subset \lambda^{(1)} \subset \lambda^{(2)} \subset \cdots \subset \lambda^{(m)}\right)$ with $\lambda^{(m)}=\lambda$ and a vector $c^{*}=\left(c_{1}, c_{2}, \ldots, c_{m}\right)$ where $c_{i}$ is the content of the cell of $S^{*}$ containing $i^{*}$. In this case, we will say the rank of $S^{*}$ is $m$.

Definition 6.1. Let $S^{*}=\left(S, c^{*}\right)$ be a starred strong tableau of rank $m$. Fix $1<i<m$. Consider the locations of $(i-1)^{*}, i^{*},(i+1)^{*}$ in $S^{*}$. The $i$-witness, or simply the witness when $i$ is fixed, is chosen among $\{i-1, i, i+1\}$ as follows.

1. If $c_{i-1} \neq c_{i+1}$, then $c_{i-1}, c_{i}, c_{i+1}$ are all distinct since consecutive ribbons cannot have heads or tails of the same content by the analysis in Section 5.2. In this case, the witness is the index of the median of the set $\left\{c_{i-1}, c_{i}, c_{i+1}\right\}$.

2. If $c_{i-1}=c_{i+1}$, then we have three cases to consider.

(a) If the $(i-1)$-ribbons and $(i+1)$-ribbons have the same length, then $i+1$ is the witness. 

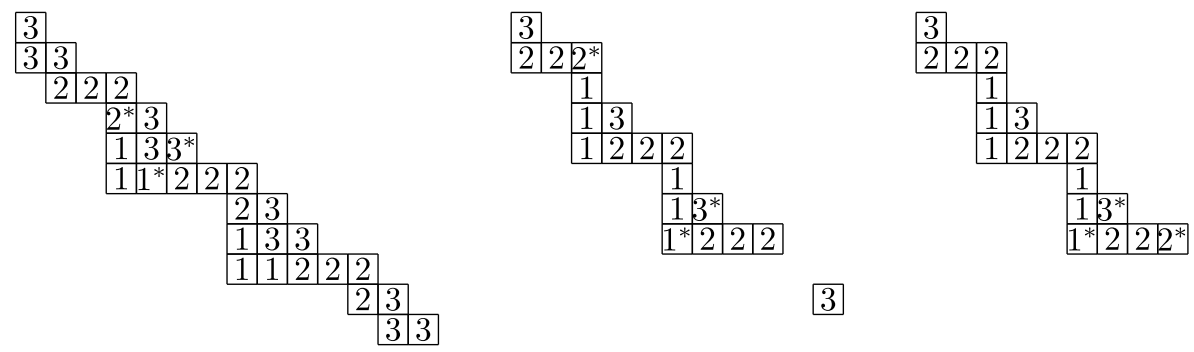

3

3

Figure 6: The witnesses from left to right are 3,1,3 demonstrating parts 2(a), $2(\mathrm{~b})$ and $2(\mathrm{c})$ respectively in Definition 6.1.

(b) If the $(i-1)$-ribbons and $(i+1)$-ribbons have different lengths and $c_{i-1}>c_{i}$, then the witness is the letter indexing the longer ribbons among the $(i-1)$-ribbons and the $(i+1)$-ribbons.

(c) If the $(i-1)$-ribbons and $(i+1)$-ribbons have different lengths and $c_{i-1}<c_{i}$, then the witness is the letter indexing the shorter ribbons among the $(i-1)$-ribbons and the $(i+1)$-ribbons.

In Figure 6, we give three examples where the witness is computed using Part (2) of Definition 6.1. Note, $i-1, i, i+1$ have been replaced by $1,2,3$ in the examples to emphasize that we only care about 3 consecutive values when computing the witness. The exact value of $i$ is irrelevant.

Note that when $S^{*}$ is a Young tableau, the contents of the unique cells containing $i-1, i$ and $i+1$ must all be distinct, ensuring that the witness is always the index of the median of the set $\left\{c_{i-1}, c_{i}, c_{i+1}\right\}$.

Next we define the involution $\varphi_{i}$ on starred strong tableaux that will serve as a model for dual equivalence. Intuitively, if $i$ and $j$ are witnessed by $h$ in $S^{*}$, then an elementary dual equivalence move should be based on the map $\operatorname{swap}_{i, j}$ where $\{h, j\}=\{i-1, i+1\}$. This would be straightforward but for the difficulty of defining how the stars should behave under such an action. We obtained these rules experimentally guided by the principle that the stars should move as little as possible while preserving the spin statistic, always remaining in the same connected component of the union of cells in $S^{*}$ containing $i-1, i, i+1$ but necessarily switching which letter they adorn whenever $i \neq h$. This will be the action of $\varphi_{i}$ whenever such a move is possible without changing the witness. However, if the interval is a chain and the starred letters both lie in the same connected component, then neither an interval swap nor a star swap is possible. We overcome this challenge by exchanging saturated chains of length three. 
Definition 6.2. Fix a starred strong tableau $S^{*}=\left(S, c^{*}\right)$ of rank $m$ with $1<i<m$. Let $h$ be the $i$-witness for $S^{*}$. If $h \neq i$, then let $j$ be defined by $\{i-1, i+1\}=\{j, h\}$. Let $S_{q}$ be the union of all $q$-ribbons and let $S_{q^{*}}$ be the connected component of $S_{q}$ containing $q^{*}$ for $1 \leq q \leq m$. We will say $S_{q}$ nests $S_{p^{*}}$ if the content of every cell of $S_{p^{*}}$ is also the content of a cell in $S_{q}$ but no head or tail of a ribbon in $S_{q}$ has the same content as the head or tail of $S_{p^{*}}$. Similarly, a connected skew shape $A$ nests another connected skew shape $B$ provided the content of every cell of $B$ is the content of some cell of $A$, but the largest and smallest contents of cells in $A$ are not the contents of any cells in $B$. Let $b_{q}$ be the content of the ribbon tail for $S_{q^{*}}$. We will say $S_{i}$ and $S_{j}$ are not abutting if $b_{i}, b_{j},\left(c_{i}+1\right),\left(c_{j}+1\right)$ have distinct residues, otherwise $S_{i}$ and $S_{j}$ are abutting. Let $B_{i}$ and $B_{j}$ be the connected components of $S_{i} \cup S_{j}$ containing $i^{*}$ and $j^{*}$, respectively.

Then $\varphi_{i}\left(S^{*}\right)$ is defined by the first case that applies below. N.B. The order matters.

$$
\varphi_{i}\left(S^{*}\right)= \begin{cases}S^{*} & \text { if } i=h, \\ \operatorname{bswap}_{i, j}\left(S^{*}\right) & \text { if } S_{i} \text { and } S_{j} \text { are not abutting, } \\ \operatorname{bswap}_{i, j}\left(S^{*}\right) & \text { if } B_{i} \text { and } B_{j} \text { have different shapes } \\ & \text { and neither nests } S_{h^{*}}, \\ \operatorname{snake}_{i, j}^{h}\left(S^{*}\right) & \text { if } b_{h} \equiv b_{j} \text { and } c_{h} \equiv c_{j}, \\ \operatorname{bswap}_{i, j} \mathrm{bswap}_{i, h}\left(S^{*}\right) & \text { if } S_{i} \text { or } S_{j} \text { nests } S_{h^{*}}, \\ \operatorname{double}_{i, j}^{h}\left(S^{*}\right) & \text { if } B_{i} \text { or } B_{j} \text { nests } S_{h^{*}}, \\ \operatorname{star}_{i, j}\left(S^{*}\right) & \text { if } B_{i} \neq B_{j} \text { but they have } \\ & \text { the same shape. }\end{cases}
$$

Here the map $\varphi_{i}$ depends on four types of ribbon swaps: basic swap, snake swap, double swap, and star swap. Each of these maps are defined below with an example on starred strong tableaux. One can already observe that for every starred strong tableau of rank $m$ at least one of the conditions for $\varphi_{i}$ must be met whenever $1<i<m$.

Note that the ribbon swaps will only be well-defined under certain circumstances. We prove, in a series of lemmas following the definitions, that the circumstances where a ribbon swap is applied in (6.1) will be precisely the circumstances when the ribbon swap is well-defined and $\varphi_{i}$ acts as an 
involution. The lemmas then immediately imply Theorem 6.12 , which says that $\varphi_{i}: \operatorname{SST}^{*}(\lambda / \mu, n) \rightarrow \operatorname{SST}^{*}(\lambda / \mu, n)$ is a well-defined involution. To show $\varphi_{i}$ is an involution, it is essential to keep track of the witness $h^{*}$. We advise the reader to pay careful attention to the witness in each of the ribbon swap definitions and examples.

The basic swap, denoted $\operatorname{bswap}_{i, j}\left(S^{*}\right)$, is the result of an interval swap on $S$ and interchanging the blocks containing $i^{*}$ and $j^{*}$

$$
\operatorname{bswap}_{i, j}\left(S^{*}\right)=\left(\operatorname{swap}_{i, j}(S), c^{*}\left(B_{i} \leftrightarrow B_{j}\right)\right) .
$$

For example, if $n=4$ and $i=4$ then $\varphi_{4}=\operatorname{bswap}_{4,5}$ interchanges

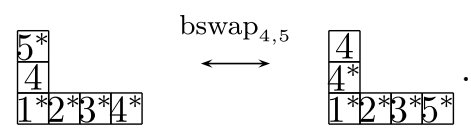

In the left tableau, $B_{4}$ is the cell of content 3 filled by $4^{*}$ and $B_{5}$ is the set of cells with contents $\{-1,-2\}$ filled by $4,5^{*}$. In the right tableau $B_{5}$ is the cell of content 3 and $B_{4}$ is the set of cells with contents $\{-1,-2\}$. Note, the star in the $\{-1,-2\}$ block must move when applying the map in either direction so as to return a valid starred strong tableau with a star at the head of an $i$-ribbon and a $j$-ribbon.

A description of the operation $c^{*}\left(B_{i} \leftrightarrow B_{j}\right)$ is given specifically as follows. Let $d_{p}=c_{p}+1$ for each $p$ so that the $p$-ribbons in $S^{*}$ correspond with applying the transposition $t_{b_{p}, d_{p}}$. Let $r_{p}=d_{p}-b_{p}$ be the length of a $p$-ribbon in $S^{*}$. Let $\varepsilon_{p}$ be the unit vector with a 1 in the $p$-th position. Assume $p<q$, then define

$$
\operatorname{flop}_{q, p}\left(c^{*}\right)=\operatorname{flop}_{p, q}\left(c^{*}\right)= \begin{cases}t_{p, q}\left(c^{*}\right)-r_{p} \cdot \varepsilon_{p} & \text { if } d_{p} \equiv d_{q} \text { and }\left|B_{p}\right|<\left|B_{q}\right|, \\ t_{p, q}\left(c^{*}\right)-r_{q} \cdot \varepsilon_{q} & \text { if } d_{p} \equiv d_{q} \text { and }\left|B_{p}\right|>\left|B_{q}\right|, \\ t_{p, q}\left(c^{*}\right)+r_{q} \cdot \varepsilon_{q} & \text { if } b_{q} \equiv d_{p} \text { and }\left|B_{p}\right|>\left|B_{q}\right|, \\ t_{p, q}\left(c^{*}\right)+r_{p} \cdot \varepsilon_{p} & \text { if } b_{p} \equiv d_{q} \text { and }\left|B_{p}\right|<\left|B_{q}\right|, \\ t_{p, q}\left(c^{*}\right) & \text { otherwise. }\end{cases}
$$

Therefore, formally we define

$$
\operatorname{bswap}_{i, j}\left(S^{*}\right)=\left(\operatorname{swap}_{i, j}(S), \text { flop }_{i, j}\left(c^{*}\right)\right) .
$$

We prove $\operatorname{bswap}_{i, j}\left(S^{*}\right)$ is always a valid starred strong tableau in Lemmas 6.5 and 6.6. 
Remark 6.3. Observe that $\operatorname{flop}_{i, j}\left(c^{*}\right)=t_{i, j}\left(c^{*}\right.$ if and only if $S_{i}$ and $S_{j}$ are not abutting. It might seem easier to remove this case from the definition of a $\mathrm{flop}_{i, j}$, however, that would add extra cases later when we discuss the case $S_{i}$ or $S_{j}$ nests $S_{h^{*}}$.

Remark 6.4. Note that when $S^{*}$ is a Young tableau, it is impossible for the cell containing $i$ to abut the cell containing $j$ when $h \neq i$ is the witness. Therefore the required ribbon swap in this case will always be $\varphi_{i}\left(S^{*}\right)=$ $\operatorname{bswap}_{i, j}\left(S^{*}\right)=\left(\operatorname{swap}_{i, j}(S), t_{i, j}\left(c^{*}\right)\right)$. Hence $\varphi_{i}$ reduces to the usual elementary dual equivalence relation on Young tableaux.

The snake swap, denoted snake ${ }_{i, j}^{h}\left(S^{*}\right)$, is the result of moving the stars on all three ribbons $i-1, i, i+1$ while keeping the underlying strong tableau fixed. If $i-1$ is the witness, the moves are based on the permutation $231=$ $t_{12} t_{23}$; if $i+1$ is the witness, the moves are based on the permutation $312=$ $t_{23} t_{12}$. Either way, $j$ will become the $i$-witness of snake $\mathrm{e}_{i, j}^{h}\left(S^{*}\right)$. Assuming $h$ is the witness, then

$$
\text { snake }_{i, j}^{h}\left(S^{*}\right)= \begin{cases}\left(S, t_{i, j} t_{i, h}\left(c^{*}\right)-r_{j} \cdot \varepsilon_{i}+r_{h} \cdot \varepsilon_{h}\right) & \text { if }\left(c_{j}<c_{i}\right) \text { xor }(i<j), \\ \left(S, t_{i, j} t_{i, h}\left(c^{*}\right)+r_{i} \cdot \varepsilon_{i}-r_{i} \cdot \varepsilon_{h}\right) & \text { otherwise. }\end{cases}
$$

We will show in the proof of Theorem 6.12 that snake $e_{i, j}^{h}$ is only applied when $S_{i} \cup S_{j}$ and $S_{i} \cup S_{h}$ are both single connected ribbons so $\left[\lambda^{(i-2)}, \lambda^{(i+1)}\right]$ is a chain by Proposition 5.11. When $h=i+1$, the stars move away from the diagonal of content $c_{h}$ along these ribbons and when $h=i-1$ the stars move in toward the diagonal of content $c_{h}$ along these ribbons. The star on the witness toggles between $h$ and $j$ by sliding along the diagonal with content $c_{h}$. For example, if $n=2$ and $i=3$, then $\varphi_{3}=$ snake $_{3,2}^{4}$ maps

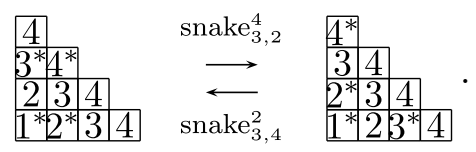

The inverse map is given by snake ${ }_{3,4}^{2}$ applied to the tableau on the right.

The double swap, denoted double ${ }_{i, j}^{h}\left(S^{*}\right)$, is the result of two interval swaps on $S$ and another "almost permutation" of the three relevant indices in the content vector. Precisely,

$$
\operatorname{double}_{i, j}^{h}\left(S^{*}\right)= \begin{cases}\left(\operatorname{swap}_{i, j} \operatorname{swap}_{i, h}(S), t_{i, j} t_{i, h}\left(c^{*}\right)+r_{h} \cdot \varepsilon_{h}\right) & \text { if } b_{h} \equiv b_{j} \\ \left(\operatorname{swap}_{i, j} \operatorname{swap}_{i, h}(S), t_{i, j} t_{i, h}\left(c^{*}\right)-r_{h} \cdot \varepsilon_{i}\right) & \text { if } c_{h} \equiv c_{j} .\end{cases}
$$


Since double ${ }_{i, j}^{h}$ is only applied when $B_{i}$ or $B_{j}$ nests $S_{h^{*}}$ but neither $S_{i}$ or $S_{j}$ nests $S_{h^{*}}$, we can conclude that the nesting block is a ribbon and that $S_{j}$ contains a cell with the same content as either the head or tail of $S_{h^{*}}$ by considering all possible rank 2 abutting rod exchanges. Thus, when its applied either $b_{h} \equiv b_{j}$ or $c_{h} \equiv c_{j}$. For example, if $n=3$ and $i=4$, then $\varphi_{4}$ interchanges the following tableaux via double swaps:

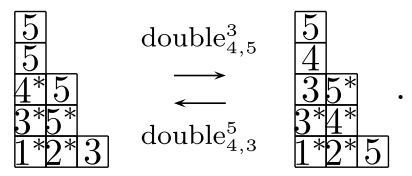

The star swap, denoted $\operatorname{star}_{i, j}\left(S^{*}\right)$, is the result of moving the star on $i^{*}$ to the adjacent $j$-ribbon and vice versa while keeping the underlying strong tableau fixed. To be precise, if $B_{i}$ and $B_{j}$ are distinct and both $B_{i}$ and $B_{j}$ contain both an $i$ and $j$-ribbon, then both blocks have the same shape by Proposition 5.10 and Proposition 5.11. Say $f$ is the offset of the contents of $B_{j}$ from $B_{i}$, so $c_{i}+f$ is the content of the head of the $i$-ribbon in $B_{j}$ and $c_{j}-f$ is the content of the head of the $j$-ribbon in $B_{i}$. Then

$$
\operatorname{star}_{i, j}\left(S^{*}\right)=\left(S, c^{*}+f \cdot \varepsilon_{i}-f \cdot \varepsilon_{j}\right) .
$$

For example, if $n=4$ and $i=6$, then $\varphi_{6}=\operatorname{star}_{6,7}$ interchanges

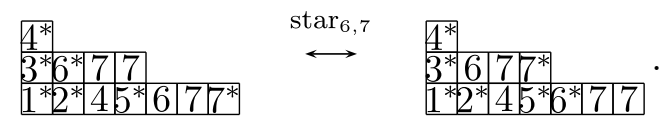

\subsection{A well-defined involution}

Given the complicated definition of the affine dual equivalence relations, it is not obvious that $\varphi_{i}$ is well-defined, much less that it is an involution. Our next task is to establish these two facts. In the course of doing so, we provide many more examples of the action of $\varphi_{i}$, though in the interest of space only the relevant cells in the strong tableaux are shown. Since the argument involves many details, it may comfort the reader to know that the conclusion of this subsection Theorem 6.12 has also been verified by computer using the techniques from Section 7.

For each of the following lemmas and proofs, we assume all of the notation from Definition 6.2. In particular, assume $1<i<m$, and let $S^{*}$ be a starred strong tableau with $i$-ribbons in the skew shape $S_{i}, j$-ribbons in the 
shape $S_{j}$, the block $B_{i}$ is the connected component of $S_{i} \cup S_{j}$ containing $i^{*}$, etc. We can assume $i \neq h$ throughout the proofs, otherwise $\varphi_{i}\left(S^{*}\right)=S^{*}$ by definition.

Lemma 6.5. Assume $i \neq h$ and that $S_{i}$ and $S_{j}$ are not abutting. Then $\operatorname{bswap}_{i, j}\left(S^{*}\right)$ is a valid starred strong tableau that satisfies the same assumptions and $\operatorname{bswap}_{i, j} \operatorname{bswap}_{i, j}\left(S^{*}\right)=S^{*}$.

Proof. In this case, a $\operatorname{swap}_{i, j}(S)$ is well defined and $b_{i}, b_{j}, d_{i}, d_{j}$ are all distinct $\bmod n$ by the classification of rod exchanges for rank 2 intervals in Section 5.2. Unless $S_{i}$ and $S_{j}$ come from an interleaving rod exchange with some $i$-ribbon nested in an $i+1$-ribbon or vice versa, the interval swap will simultaneously change all $i$ 's to $j$ 's and conversely. Therefore $\varphi_{i}\left(S^{*}\right)=\operatorname{bswap}_{i, j}\left(S^{*}\right)=\left(\operatorname{swap}_{i, j}(S), t_{i, j}\left(c^{*}\right)\right)$ is a well-defined starred strong tableau with stars in the original cells in $S^{*}$, though now adorning the opposite letter among $\{i, j\}$ from before. When $S_{i}$ and $S_{j}$ come from an interleaving rod exchange with some $i$-ribbon nested in an $i+1$-ribbon or vice versa, then the interval swap will change all entries in the shorter ribbon appearing independently as well as entries in the longer ribbon not on the same content as a shorter ribbon. In particular, the shape of the blocks $B_{i}$ and $B_{j}$ remains unchanged. Therefore $\operatorname{bswap}_{i, j}\left(S^{*}\right)$ is again a valid starred strong tableau. In this case, the star adorning the longer ribbon remains in place, and the star adorning the shorter ribbon remains if the shorter ribbon is not nested in a longer, otherwise it slides one position along the diagonal; see Figure 7 for an example.

Consequently, in order to show $\varphi_{i}$ is an involution in this case, it remains only to show that $h$ remains the witness after applying $\operatorname{bswap}_{i, j}$. Since the effect on the content vector is merely to interchange $c_{i}$ and $c_{j}$, the result follows provided $c_{h} \neq c_{j}$. However, the contrary case forces an $i$-ribbon to abut both the $i-1$-ribbon and $i+1$-ribbon with heads on content $c_{i-1}=c_{i+1}$. This would force the tail of the $i$-ribbons to be on the next diagonal in order to create a valid skew shape, contradicting the assumption that $S_{i}$ and $S_{j}$ are not abutting. Hence, bswap $_{i, j}$ is an involution in this case.

Henceforth, we can assume that $S_{i}$ and $S_{j}$ are abutting, and thus both $B_{i}$ and $B_{j}$ must have ribbon shape by Corollary 5.12.

Lemma 6.6. Assume $i \neq h$ and that $S_{i}$ and $S_{j}$ are abutting. Further assume that $B_{i}$ and $B_{j}$ have different ribbon shapes but neither nests $S_{h^{*}}$. Then $T^{*}=$ $\operatorname{bswap}_{i, j}\left(S^{*}\right)$ is a starred strong tableau that satisfies the same assumptions and $\operatorname{bswap}_{i, j}\left(T^{*}\right)=S^{*}$. 


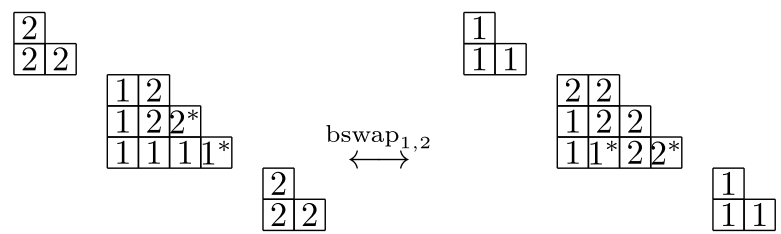

Figure 7: The action of $\varphi_{i}$ when $S_{i}$ and $S_{j}$ are nested, hence not abutting.
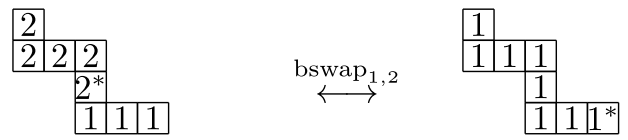

$1|1| 1 *$

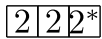

Figure 8: The action of $\varphi_{i}$ when $S_{i} \cup S_{j}$ is abutting and $S_{h}$ is not nested.

Proof. Since $B_{i}$ and $B_{j}$ have different shapes, the map swap $i, j$ will toggle between each block containing only one letter and exactly one of these blocks containing both letters as shown in Figure 8. By Proposition 5.10 one can deduce how the stars move in the blocks $B_{i}$ and $B_{j}$ in order to adorn the other letter. These moves are summarized in the function $\operatorname{flop}_{i, j}\left(c^{*}\right)$. Hence, $T^{*}=\varphi_{i}\left(S^{*}\right)=\operatorname{bswap}_{i, j}\left(S^{*}\right)$ is a well defined starred strong tableau. Furthermore, by inspection we have that $\operatorname{bswap}_{i, j}\left(T^{*}\right)=S^{*}$. Thus, $\varphi_{i}\left(S^{*}\right)$ is an involution provided $h$ is also the $i$-witness of $T^{*}$.

Observe that the only way for the witness to change is if $h^{*}$ lies on a diagonal within a block containing both $i$ 's and $j$ 's, and $h^{*}$ lies weakly between their respective heads. Let $I$ and $J$ be the abutting $i$-ribbon and $j$-ribbon in the block overlapping $h^{*}$. By Proposition 5.8, consecutive ribbons may not have partially overlapping contents. Therefore if an $h$-ribbon has content overlapping an $i$-ribbon, one of the two must be nested. By assumption, $S_{h^{*}}$ is not nested inside either $B_{i}$ or $B_{j}$, hence is not nested in $I$. On the other hand, if $h$-ribbons nest $i$-ribbons, then they must also nest $j$-ribbons, otherwise a $\operatorname{swap}_{i, h}(S)$ is possible and will leave $i$-ribbons and $j$-ribbons with partially overlapping contents, again contradicting Proposition 5.8. Therefore $h$-ribbons and $i$-ribbons may not have overlapping contents, so the cell containing $h^{*}$ must overlap $J$ in content.

If $J$ lies southeast of $I$, this forces the $h^{*}$-ribbon to overlap $I$ or be nested in $J$, neither of which is possible. Thus $J$ must lie northwest of $I$, hence the head of the $h$-ribbon is forced to have the same content as the head of $J$. Furthermore, the $h^{*}$-ribbon must be longer than $J$ since by 

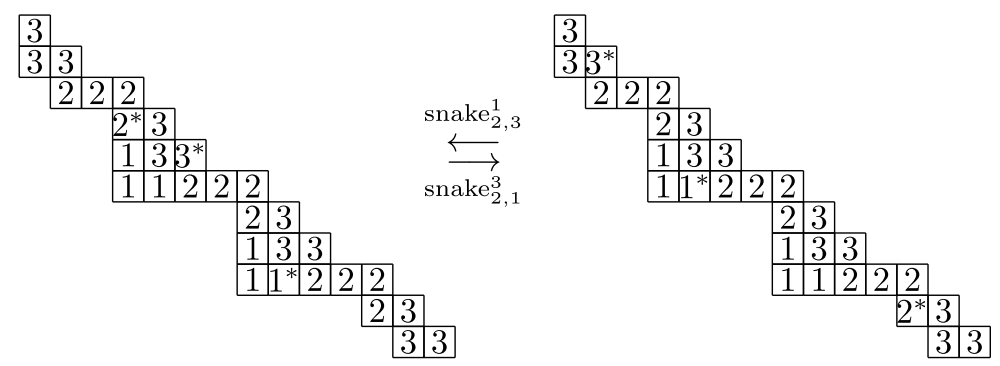

Figure 9: The action of $\varphi_{i}$ when $i-1$-ribbons and $i+1$-ribbons share a head and tail.

assumption $h$-ribbons are not nested in $I \cup J$. Therefore when $h^{*}$ and $j^{*}$ lie on the same diagonal, $h$ remains the $i$-witness for $\varphi_{i}\left(S^{*}\right)$ using part (2) of Definition 6.1.

Lemma 6.7. Assume $i \neq h$ and that $c_{h} \equiv c_{j}$ and $b_{h} \equiv b_{j}$. Then $\operatorname{snake}_{i, j}^{h}\left(S^{*}\right)$ is a well-defined starred strong tableau that satisfies the same assumptions with the roles of $h$ and $j$ reversed and snake $_{i, h}^{j}\left(\operatorname{snake}_{i, j}^{h}\left(S^{*}\right)\right)=S^{*}$.

Proof. Recall the notation $d_{p}=c_{p}+1$ so that the $p$-ribbons in $S^{*}$ correspond with applying the transposition $t_{b_{p}, d_{p}}$ for each $p$. By the assumptions then, $t_{b_{i-1}, d_{i-1}}=t_{b_{i+1}, d_{i+1}}$ as affine permutations. By Corollary $5.6, S^{*}$ is associated to a transposition sequence $\left(t_{b_{1} d_{1}} \rightarrow t_{b_{2} d_{2}} \rightarrow \cdots \rightarrow t_{b_{m} d_{m}}\right)$. In order for $t_{b_{i-1} d_{i-1}} \rightarrow t_{b_{i} d_{i}} \rightarrow t_{b_{i+1} d_{i+1}}$ to be a valid consecutive triple in the transposition sequence, $t_{b_{i} d_{i}}$ must not commute with the other two. Hence at least one $i+1$-ribbon completely overlaps some $i$-1-ribbon, sharing both a head and tail, and $i$-ribbons must abut each such pair from both sides. By Proposition 5.11, this means $S_{i} \cup S_{j}$ and $S_{i} \cup S_{h}$ are both ribbons, hence snake $\mathrm{i}_{i, j}^{h}$ is well-defined on $S^{*}$.

By inspecting (6.4), we see that $T^{*}=\operatorname{snake}_{i, j}^{h}\left(S^{*}\right)$ is a starred strong tableau on the same underlying strong tableau $S$ with $j$ as its $i$-witness, so $\varphi_{i}\left(T^{*}\right)=\operatorname{snake}_{i, h}^{j}\left(T^{*}\right)=S^{*}$. For example, see Figure 9.

Henceforth, we will assume that $S_{i}$ and $S_{j}$ are abutting and either $c_{h} \not \equiv c_{j}$ or $b_{h} \not \equiv b_{j}$. The next case to consider is when $B_{i}$ or $B_{j}$ nests $S_{h^{*}}$, including the possibility that $S_{i}$ or $S_{j}$ nests $S_{h^{*}}$. Note that if $B_{i}=B_{j}$, then $B_{i}$ necessarily nests $S_{h^{*}}$ in order for $h$ to be the witness. The following technical lemma will be useful.

Lemma 6.8. Assume $i \neq j, S_{i}$ and $S_{j}$ are abutting, either $c_{h} \not \equiv c_{j}$ or $b_{h} \not \equiv$ $b_{j}$, and $B_{i}$ or $B_{j}$ nests $S_{h^{*}}$. Then, some connected component of $S_{h} \cup S_{i} \cup S_{j}$ is a single $h$-ribbon. 
Proof. If some $h$-ribbon is nested in an $i$-ribbon, then the claim follows immediately from Lemma 5.9. Similarly, one can also show that if some $h$-ribbon is nested in a $j$-ribbon, then the claim follows by examining the relative rod lengths in the exchanges. So, assume that some connected component of $S_{i} \cup S_{j}$ contains both $i$ 's and $j$ 's and nests an $h$-ribbon. By Proposition 5.8, we may further assume that the nested $h$-ribbon shares a head or tail with the $j$-ribbon. Necessarily the $h$-ribbon and $j$-ribbon must both abut an $i$-ribbon on the diagonal of shared content in the strong tableau. Therefore we have one of the following two types of transposition sequences corresponding to the $i-1, i, i+1$ ribbons in $S^{*}$, drawn schematically assuming $a<b<c<d \leq a+n$.
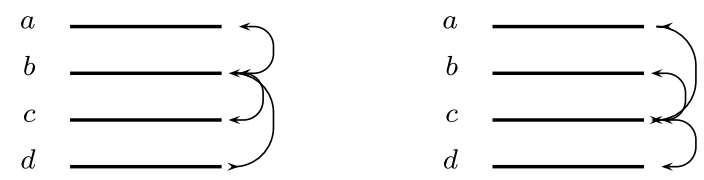

To ease notation we assume $h=i-1$ and $j=i+1$ and we are doing the rod exchange on the left in (6.5), noting that the other cases are completely analogous. In this case, analyzing the transposition sequence $t_{b, c} \rightarrow t_{a, b} \rightarrow$ $t_{b, d}$ shows that initially the length of $\operatorname{rod}(b)$ cannot be weakly between the lengths of $\operatorname{rod}(a)$ and $\operatorname{rod}(d)$ or else the transpositions don't each increase the rank by exactly one at each step. Furthermore, the length of $\operatorname{rod}(b)$ cannot be less than the length of $\operatorname{rod}(d)$ because otherwise there would be no $i+1$-ribbon with content overlapping any $i-1$-ribbon by the definition of a transposition sequence from Definition 5.5 and Corollary 5.6 contradicting the assumption that some connected component of $S_{i} \cup S_{j}$ contains both $i$ 's and $j$ 's and nests an $h$-ribbon. Therefore, the length of $\operatorname{rod}(b)$ is strictly greater than the length of $\operatorname{rod}(a)$ so, by Corollary 5.6 again, there must an $i$-1-ribbon occurring independently from all $i, i+1$-ribbons.

Lemma 6.9. Assume $i \neq h$, that $S_{i}$ and $S_{j}$ are abutting, and either $c_{i-1} \not \equiv$ $c_{i+1}$ or $b_{i-1} \not \equiv b_{i+1}$. Further assume that $S_{i}$ or $S_{j}$ nests $S_{h^{*}}$. Then $U^{*}=$ $\varphi_{i}\left(S^{*}\right)=\operatorname{bswap}_{i, j} \operatorname{bswap}_{i, h}\left(S^{*}\right)$ is a starred strong tableau that satisfies the same assumptions except with the roles of $j$ and $h$ reversed. Furthermore, $\varphi_{i}\left(U^{*}\right)=S^{*}$.

Proof. By Lemma 6.8, we know some $h$-ribbon appears independently from all $i$-ribbons. So by Proposition 5.11, the interval corresponding to the $i, h$ ribbons in the $n$-core poset is not a chain. Therefore, $T=\operatorname{swap}_{i, h}(S)$ is a valid strong tableau. Similarly, in $T$, some $i$-ribbon appears independently of all $j$-ribbons, making $U=\operatorname{swap}_{i, j}(T)$ well defined. In each case, the swap 
is just applying a nested ribbon exchange. Thus, we observe that $T^{*}=$ $\operatorname{bswap}_{i, h}\left(S^{*}\right)$ is a valid starred strong tableau. Similarly, some $i$-ribbon in $T^{*}$ appears independently of all $j$-ribbons in $T^{*}$, making $U^{*}=\operatorname{bswap}_{i, j}\left(T^{*}\right)$ well defined. The contents of the stars on $h, i, j$, will simply be permuted since each basic swap is just applying a nested ribbon exchange. Therefore, in $U^{*}$ the $i$-witness is $j, S_{i}$ and $S_{h}$ are abutting and $S_{h}$ or $S_{i}$ must nest $S_{j^{*}}$ so $\varphi_{i}$ applied to $U^{*}$ is applied according to the same case as $S^{*}$. Since bswap bi,j $_{\text {is }}$ an involution when it can be applied, it clearly follows that $\varphi_{i}\left(U^{*}\right)=S^{*}$.

Lemma 6.10. Assume $i \neq h$, that $S_{i}$ and $S_{j}$ are abutting, and either $c_{i-1} \not \equiv$ $c_{i+1}$ or $b_{i-1} \not \equiv b_{i+1}$. Further assume that $B_{i}$ or $B_{j}$ nests $S_{h^{*}}$ but neither $S_{i}$ nor $S_{j}$ nests $S_{h^{*}}$. Then $U^{*}=$ double $_{i, j}^{h}\left(S^{*}\right)$ is a valid starred strong tableau that satisfies the same assumptions except with the roles of $j$ and $h$ reversed. Furthermore, double ${ }_{i, h}^{j}\left(U^{*}\right)=S^{*}$.

Proof. Similar to the proof of Lemma 6.9, we know that both $T=\operatorname{swap}_{i, h}(S)$ and $U=\operatorname{swap}_{i, j}(T)$ are valid strong tableaux. However, each of these swaps are abutting exchanges instead of nested exchanges. The final result of $\operatorname{swap}_{i, j}\left(\operatorname{swap}_{i, h}(S)\right)$ is much like a single interval swap in the case of nested $i, i+1$-ribbons: all independently occurring $h$ 's change to $j$ 's and all letters of $S_{i} \cup S_{j}$ not on the same diagonal as an $h$ will change with $i$ 's becoming $h$ 's and $j$ 's becoming $i$ 's; for example, see Figure 10. The shape and contents of the nested ribbon remains the same, but these are $j$-ribbons in $\operatorname{swap}_{i, j}\left(\operatorname{swap}_{i, h}(S)\right)$. Therefore, $U^{*}=\operatorname{double}_{i, j}^{h}(S)$ is a well-defined starred strong tableau with a star placed at the head of some $p$-ribbon for each $p$. The effect of $\varphi_{i}$ on the content vector for $S^{*}$ is an involution by inspection. Since $j$-ribbons are now nested in $U^{*}$, we only need to show $j$ becomes the $i$-witness in $U^{*}$ in order to prove $\varphi_{i}$ is an involution on such an $S^{*}$. This will clearly be the case so long as $c_{h} \neq c_{j}$ both before and after applying double $_{i, j}^{h}$. Assuming $c_{h}=c_{j}$, an $i$-ribbon will be forced to lie southeast of $S_{h^{*}}$ and $S_{j^{*}}$ by the definition of the witness. However, after applying $\varphi_{i}\left(S^{*}\right), h$ ribbons and $j$-ribbons will share a tail instead, so the witness indeed changes as desired.

Lemma 6.11. Assume $i \neq h$, that $S_{i}$ and $S_{j}$ are abutting, and either $c_{i-1} \not \equiv$ $c_{i+1}$ or $b_{i-1} \not \equiv b_{i+1}$. Further assume that $B_{i}$ and $B_{j}$ have the same shape but lie on distinct content diagonals, each have ribbon shape, and neither nests $S_{h^{*}}$. Then $T^{*}=\operatorname{star}_{i, j}\left(S^{*}\right)$ is a valid starred strong tableau that satisfies the same assumptions. Furthermore $\operatorname{star}_{i, j}\left(T^{*}\right)=S^{*}$.

See Figure 11 for an example. 


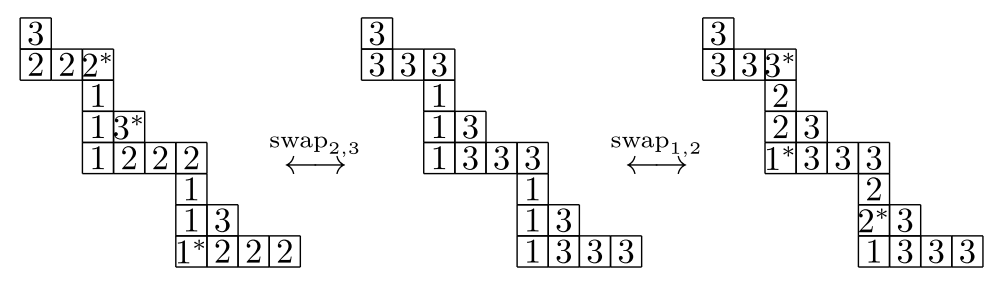

3

1

Figure 10: The action of $\varphi_{2}=$ double $_{i, j}^{h}$ when $S_{i^{*}} \cup S_{j^{*}}$ nests $S_{h^{*}}$.

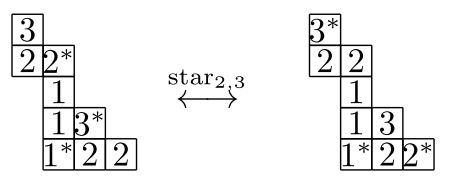

Figure 11: The action of $\varphi_{i}$ when $h^{*}$ overlaps $S_{i^{*}} \cup S_{j^{*}}$ without being nested.

Proof. The result of a star swap is clearly a valid starred strong tableau. We only need to prove that the $i$-witness of $T^{*}$ is still $h$ so it satisfies the same conditions when $\varphi_{i}$ is applied, proving it is an involution.

Since blocks $B_{i}$ and $B_{j}$ have the same shape by assumption, both blocks contain $i$ 's and $j$ 's. The only way for the witness to change is if $h^{*}$ lies on a diagonal within a block containing both $i$ 's and $j$ 's, and $h^{*}$ lies weakly between their respective heads. The proof that $h$ remains the witness is the same as the argument at the end of the proof of Lemma 6.10.

We have covered every case in the definition of $\varphi_{i}$ from (6.1). The following theorem now follows directly from the previous 7 lemmas.

Theorem 6.12. For each $1<i<m$, the map $\varphi_{i}$ is a well-defined involution on all starred strong tableaux of a fixed $n$-core $\lambda$ of rank $m$.

Remark 6.13. The cases in (6.1) partition the set of all starred strong tableaux into 7 distinct $\varphi_{i}$-types. From the lemmas, we observe that the type of $S^{*}$ and $\varphi_{i}\left(S^{*}\right)$ is always the same.

\subsection{Preservation of spin}

Next we show that the involution $\varphi_{i}$ has the added feature of preserving the spin statistic. Recall from (3.2) that spin is defined by

$$
\operatorname{spin}\left(S^{*}\right)=\sum_{i} n(i) \cdot(h(i)-1)+d\left(i^{*}\right),
$$


where $n(i)$ is the number of $i$-ribbons, $h(i)$ is the height of an $i$-ribbon and $d\left(i^{*}\right)$ is the depth of the starred $i$-ribbon. We will show that $\varphi_{i}$ preserves the spin by tracking the contribution for $i-1, i$ and $i+1$.

Proposition 6.14. For any starred strong tableau $S^{*}$, we have $\operatorname{spin}\left(\varphi_{i}\left(S^{*}\right)\right)=$ $\operatorname{spin}\left(S^{*}\right)$.

Proof. Recall the notation from Definition 6.2. Assume $i \neq h$. If $S_{i}$ and $S_{j}$ are disjoint, interleaving or nested with non-overlapping content, then $\varphi_{i}$ acts by simultaneously replacing all $i$ 's with $j$ 's and conversely. The contribution to spin for ribbons other than $i, j$ is unchanged, and these two swap contributions, thereby preserving the statistic. If any two ribbons of $S_{i}$ and $S_{j}$ are nested with overlapping contents, then recall that $\varphi_{i}$ does not change the shape of the shorter ribbon nor the height (nor width) of the longer ribbon, and the stars remain on the same diagonals. This ensures that contributions to spin for $i$ and $j$ are exchanged, and all other contributions are unchanged.

We may now assume that $S_{i}$ and $S_{j}$ are abutting for the remainder of the proof. If $\varphi_{i}$ acts by $\operatorname{star}_{i, j}$, then this affects only the depths of $i^{*}$ and $j^{*}$. We claim $d\left(i^{*}\right)+d\left(j^{*}\right)$ is preserved since every connected component of $S_{i} \cup S_{j}$ inclusively between $B_{i}$ and $B_{j}$ have the same shape when $\operatorname{star}_{i, j}$ is applied. Hence, $\varphi_{i}$ again preserves spin.

The remaining $\varphi_{i}$-types are more complicated, so they will be covered separately in Lemmas 6.15, 6.16, 6.17, and 6.18.

Lemma 6.15. Assume $i \neq h$ and that $S_{i}$ and $S_{j}$ are abutting. Further assume that $B_{i}$ and $B_{j}$ have different ribbon shapes but neither nests $S_{h^{*}}$. Then $\varphi_{i}\left(S^{*}\right)=\operatorname{bswap}_{i, j}\left(S^{*}\right)$ and $\operatorname{spin}\left(\varphi_{i}\left(S^{*}\right)\right)=\operatorname{spin}\left(S^{*}\right)$.

Proof. Recall from the proof of Lemma 6.6 that each connected component of $S_{i} \cup S_{j}$ is a ribbon, in particular one of $B_{i}$ and $B_{j}$ is a longer ribbon containing an $n$-translate of the shorter. Let $n_{l}$ and $n_{s}$ denote the number of the longer ribbons and shorter ribbons in $S_{i} \cup S_{j}$, respectively, and let $h_{l}$ and $h_{s}$ denote their respective heights. Let $d_{l}$ be the number of longer ribbons northwest of the starred long ribbon, and similarly let $d_{s}$ denote the number of shorter ribbons northwest of the starred short ribbon.

Supposing that the connected components of $S_{i} \cup S_{j}$ each contain a unique letter, the contributions for $i$ and $j$ to spin are

$$
\begin{aligned}
& \operatorname{spin}_{S^{*}}(i)=n_{s}\left(h_{s}-1\right)+d_{s}, \\
& \operatorname{spin}_{S^{*}}(j)=n_{l}\left(h_{l}-1\right)+d_{l} .
\end{aligned}
$$


On the other hand, letting $T^{*}=\operatorname{bswap}_{i, j}\left(S^{*}\right)$, some connected component of $T_{i} \cup T_{j}$ contains both $i$ 's and $j$ 's. By Proposition 5.10, this implies that in every component containing both letters, the smaller entries are south of the larger entries if and only if the shorter ribbons appear independently to the southeast. Armed with this observation, we conclude that if the longer ribbon among $B_{i}$ and $B_{j}$ contains both $i$ 's and $j$ 's, then the contribution to spin for $i$ and $j$ in $T^{*}$ is

$$
\begin{aligned}
& \operatorname{spin}_{T^{*}}(i)=\left(n_{s}+n_{l}\right)\left(h_{s}-1\right)+\left(d_{s}+\varepsilon n_{l}\right), \\
& \operatorname{spin}_{T^{*}}(j)=n_{l}\left(h_{l}-h_{s}+(1-\varepsilon)-1\right)+d_{l},
\end{aligned}
$$

where $\varepsilon$ is 1 if the shorter ribbons appear independently southeast of the longer (equivalently, the larger entry abuts the shorter from the north), and 0 if the shorter ribbons appear independently northwest of the longer (equivalently, the smaller entry abuts the larger from the west). Noting the equality between (6.6) plus (6.7) and (6.8) plus (6.9) shows spin is once again preserved.

Lemma 6.16. Assume $i \neq h$ and that $c_{h} \equiv c_{j}$ and $b_{h} \equiv b_{j}$. Then $\varphi_{i}\left(S^{*}\right)=$ $\operatorname{snake}_{i, j}^{h}\left(S^{*}\right)$ and $\operatorname{spin}\left(\varphi_{i}\left(S^{*}\right)\right)=\operatorname{spin}\left(S^{*}\right)$.

Proof. When $\varphi_{i}$ acts by snake ${ }_{i, j}^{h}$, then the difference $\operatorname{spin}\left(S^{*}\right)-\operatorname{spin}\left(\varphi_{i}\left(S^{*}\right)\right)$ only depends on the change in depth for $h^{*}, i^{*}, j^{*}$ since both $S^{*}$ and $\varphi_{i}\left(S^{*}\right)$ have the same underlying strong tableau. Furthermore, we have that $i-1$ ribbons and $i+1$-ribbons both have length $n$ minus the length of an $i$ ribbon. In this case, there is one more $i+1$-ribbon than $i$-ribbon and one more $i$-ribbon than $i$-1-ribbon. Using the intuitive definition of snake $_{i, j}^{h}$ following (6.4) we see that moving the witness from $i-1$ to $i+1$ increases the depth of the witness by one, and similarly moving from $i+1$ to $i-1$ decreases the depth by one. As the stars on $i$ and $j$ move in or out along their respective ribbons, one star necessarily moves to an abutting ribbon joined on an east/west edge and the other star moves to abutting ribbon joined on a north/south edge. Moving a star across a north/south edge will not change the depth of the star, but moving a star across an east or west edge will increase or decrease the depth by one, respectively, canceling the contribution from moving the witness. Therefore the total contribution to spin from $i-1, i, i+1$ remains the same after applying snake $\mathrm{e}_{i, j}^{h}$.

Lemma 6.17. Assume $i \neq h$, that $S_{i}$ and $S_{j}$ are abutting, and either $c_{i-1} \not \equiv c_{i+1}$ or $b_{i-1} \not \equiv b_{i+1}$. Further assume that $S_{i}$ or $S_{j}$ nests $S_{h^{*}}$. Then $\operatorname{spin}\left(\varphi_{i}\left(S^{*}\right)\right)=\operatorname{spin}\left(S^{*}\right)$. 

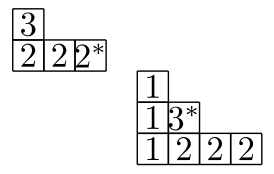

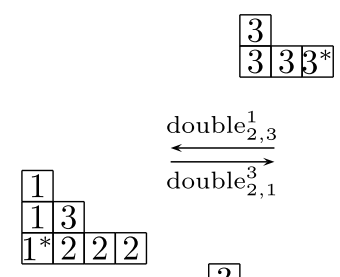

3

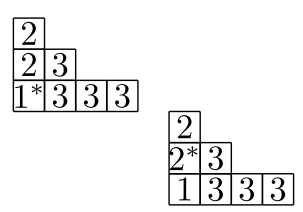

Figure 12: An example of $\varphi_{i}$ acting via $\varphi_{i}=$ double $_{i, j}^{h}$.

Proof. This follows from Lemma 6.15 where bswap $_{i, j}$ is shown to preserve spin.

Lemma 6.18. Assume $i \neq h$, that $S_{i}$ and $S_{j}$ are abutting, and either $c_{i-1} \not \equiv$ $c_{i+1}$ or $b_{i-1} \not \equiv b_{i+1}$. Further assume that $B_{i}$ or $B_{j}$ nests $S_{h^{*}}$ but neither $S_{i}$ nor $S_{j}$ nests $S_{h^{*}}$. Then $\varphi_{i}\left(S^{*}\right)=\operatorname{double}_{i, j}^{h}\left(S^{*}\right)$ and $\operatorname{spin}\left(\varphi_{i}\left(S^{*}\right)\right)=\operatorname{spin}\left(S^{*}\right)$.

Proof. We may assume, from the analysis in Lemma 6.10, that some $h$ ribbon and $j$-ribbon share a head or tail. Also from Lemma 6.10, some $h$ ribbon must appear independently of all $i$-ribbons. Furthermore, by Proposition 5.10, if $i$ - or $j$-ribbons appear independently of the other, then they do so on the opposite side of abutting $i$ - and $j$-ribbons from $h$-ribbons. Supposing first that the combined lengths of an $i$-ribbon plus a $j$-ribbon is less than $n$, reading from northwest to southeast or from southeast to northwest one sees isolated $h$-ribbons followed by abutting $i$ - and $j$-ribbons nesting $h$-ribbons. There are then three options for what follows: isolated $j$-ribbons; isolated abutting $i$ - and $h$-ribbons; or no further $i$-, $j$ - or $h$-ribbons. For example, see Figure 12. Note that, in particular, $S^{*}$ has isolated $j$-ribbons if and only if double $\mathrm{e}_{i, j}^{h}\left(S^{*}\right)$ has isolated abutting $i$ - and $h$-ribbons.

To assess the contributions to spin, assume that $S^{*}$ has no isolated abutting $i$ - and $h$-ribbons, as in the right hand side of Figure 12 . Let $h_{w}$ and $d_{w}$ denote the height and depth of the starred $h$-ribbon, respectively, and let $n_{w}$ denote the number of isolated witness ribbons. For the example, we have $n_{w}=1, h_{w}=1, d_{w}=0$. Let $n_{l}$ be the number of $i$-ribbons, each with height $h_{l}$ and the starred one with depth $d_{l}$. Let $n_{s}$ be the number of isolated $j$-ribbons, and let $h_{s}$ and $d_{s}$ denote the height and depth, respectively, of $j$-ribbons. For the example, we have $n_{l}=2, h_{l}=2, d_{l}=1$ and $n_{s}=1$, $h_{s}=2, d_{s}=0$.

The contribution to spin from $i-1, i, i+1$ in $S^{*}$, where $S^{*}$ has no isolated abutting $i$ - and $h$-ribbons, is given by 


$$
\begin{aligned}
\operatorname{spin}_{S^{*}}(h) & =\left(n_{l}+n_{w}\right)\left(h_{w}-1\right)+d_{w}, \\
\operatorname{spin}_{S^{*}}(i) & =n_{l}\left(h_{l}-1\right)+d_{l}, \\
\operatorname{spin}_{S^{*}}(j) & =\left(n_{l}+n_{s}\right)\left(h_{s}-1\right)+d_{s} .
\end{aligned}
$$

Following the description of how double $\mathrm{e}_{i, j}^{h}$ acts on these ribbons, we may similarly compute the contributions of $i-1, i, i+1$ to the spin of $T^{*}=$ double $_{i, j}^{h}\left(S^{*}\right)$. With $h$ and $j$ defined relative to $T^{*}$, we have

$$
\begin{aligned}
\operatorname{spin}_{T^{*}}(h) & =\left(n_{l}+n_{w}+n_{s}\right)\left(h_{w}-1\right)+\left(d_{w}+\varepsilon n_{s}\right), \\
\operatorname{spin}_{T^{*}}(i) & =\left(n_{l}+n_{s}\right)\left(h_{s}-h_{w}+(1-\varepsilon)-1\right)+d_{s}, \\
\operatorname{spin}_{T^{*}}(j) & =n_{l}\left(h_{l}+h_{w}-(1-\varepsilon)-1\right)+d_{l},
\end{aligned}
$$

where, similar to before, $\varepsilon$ is 0 if the witness originally existed only to the left of abutting $i$ - and $j$-ribbons and 1 otherwise. Adding the contributions in either case miraculously yields the same result, thereby showing that the spin statistic is preserved.

If $i$-ribbons and $j$-ribbons have lengths adding to $n$, we regard the abutting $i$ - and $j$-ribbons which together nest an $h$-ribbon as abutting pairs, and the leftover $\max (i, j)$-ribbon as isolated. For example, in Figure 10, we regard the left side as having 1-ribbons abutting 2-ribbons from the west with an isolated abutting 2-ribbon and 3-ribbon to the northwest, and the right side we regard as having 2-ribbons abutting 3-ribbons from the west with an isolated 3-ribbon to the northwest. That is to say, Figure 10 is the same as Figure 12 for the purposes of calculating spin. In this case, note that $S^{*}$ has no isolated abutting $i$ - and $h$-ribbons precisely when $h=i-1$. Moreover, in this case we always have $n_{s}=1$. With this alteration, the analysis of spin is precisely as before, again showing that spin is preserved.

The results in Theorem 6.12 and Proposition 6.14 naturally extend to skew partitions as well since the proofs only involve intervals of rank 3 in the $n$-core poset.

Theorem 6.19. Let $\mu \subset \nu$ be $n$-cores of lengths $\ell(\mu)=p$ and $\ell(\nu)=q$. Then, for $p<i<q$, the map $\varphi_{i}$ is a well-defined, spin preserving, involution on all starred strong skew tableaux for $\nu / \mu$. In particular, spin is constant on affine dual equivalence classes.

\section{A graph on starred strong tableaux}

In this section, we construct a vertex-signed, edge-colored graph from our elementary affine dual equivalence map $\varphi_{i}$. The main goal of this section 
is to show that this graph is, in fact, an $\mathrm{LSP}_{2}$ graph by Definition 4.5. In order to establish this, we introduce two operations on starred strong tableaux which together show that there are only finitely many isomorphism types for 2-colored connected components. The reduction to finitely many isomorphism types can be viewed as an (incomplete) analog of the jeu de taquin algorithm for starred strong tableaux. This analogy is summarized in Remark 7.12.

Definition 7.1. For an $n$-core $\nu$, the affine dual equivalence graph $\mathcal{G}_{\nu}^{(n)}$ is the signed, colored graph with vertex set $V_{\nu}$ given by the set of all starred strong tableaux $S^{*}$ of shape $\nu$, with signature function $\sigma\left(S^{*}\right)$ obtained from the reading word on the starred letters in $S^{*}$, and for each $1<i<\ell(\nu)$, the set of $i$-colored edges, $E_{i}$, is the set of all pairs $\left\{S^{*}, \varphi_{i}\left(S^{*}\right)\right\}$ such that $S^{*} \neq \varphi_{i}\left(S^{*}\right)$. This definition also extends to skew shapes $\nu / \mu$ in the $n$-core poset. For $S^{*} \in \operatorname{SST}^{*}(\nu / \mu, n)$, let $\left[S^{*}\right]$ denote the connected component of the affine dual equivalence graph $\mathcal{G}_{\nu / \mu}^{(n)}$ containing $S^{*}$.

For example, for $n=3$ and $\mu=(5,3,1)$ the affine dual equivalence graph is shown on page 395 .

Recall that $\varphi_{i}$ is an involution which preserves the spin statistic by Theorem 6.19. In order to justify our terminology of affine dual equivalence. We want to prove that the graph induced by these involutions satisfies Axioms 1, 2, 3, 5 from Definition 4.2 and local Schur positivity on all two adjacent colored connected components. Thus each affine dual equivalence graph is $\mathrm{LSP}_{2}$. The key will be reducing local Schur positivity to a finite verification. The reduction is achieved with the help of flattening rows and squashing and/or cloning columns.

\subsection{The flattening map}

Here we define an iterative procedure to flatten an $n$-core partition down to an $m$-core partition for any $1 \leq m<n$. We will extend this procedure to starred strong tableaux in a way that commutes with the affine dual equivalence involutions.

Definition 7.2. For any $m+1$-core $\lambda$ and any $1 \leq d \leq m+1$, define $\lambda^{(d)}$ to be the unique partition associated to the binary string obtained by removing all beads and spacers with content congruent to $d$ modulo $m+1$ from the abacus of $\lambda$. In particular, $\lambda^{(d)}$ is an $m$-core.

We note that the above definition makes sense in light of Remark 5.1 and the characterization of $n$-cores in terms of the $n$-rod abacus. For example, regarding $(7,4,4,2,2)$ as a 4 -core, $(7,4,4,2,2)^{(2)}$ is the 3 -core $(6,4,2)$. 

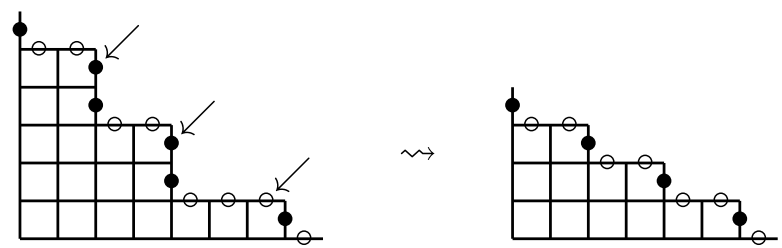

Remark 7.3. For $n$-cores $\mu \subset \nu$, if some transposition sequence from $\mu$ to $\nu$ leaves rod $d$ fixed then every transposition sequence from $\mu$ to $\nu$ leaves rod $d$ fixed. This follows from the observation that any saturated chain from $\mu$ to $\nu$ can be obtained from any other by some sequence of interval exchanges, none of which may change which rods are fixed.

Proposition 7.4. Let $\mu \subset \nu$ be $m+1$-cores such that some (equivalently, every) transposition sequence from $\mu$ to $\nu$ fixes rod $d$. Then the interval $[\mu, \nu]$ in the $m+1$-core poset is isomorphic to $\left[\mu^{(d)}, \nu^{(d)}\right]$ in the $m$-core poset. This isomorphism extends to a bijection on skew strong tableaux which preserves the number of $i$-ribbons for each $i$.

Remark 7.5. Proposition 7.4 can be used in reverse: given $\mu<\nu$ in the $m$-core poset, we can lift the interval $[\mu, \nu]$ to an isomorphic interval in the $m+1$-core posets with the same nice implications on strong tableaux. This map is implemented by using the inverse procedure of adding in an extra rod between any two existing rods. This can be done precisely when the length of the inserted rod never has length weakly between the length of two interchanging rods. For instance, we may always take the rod to be longer than all other rods or shorter than all other rods.

Proof. Since the length of rod $d$ for each $m+1$-core $\lambda$ in the interval $[\mu, \nu]$ is constant, the covering relations in the $m+1$-core poset restricted to $[\mu, \nu]$ depend on rod $d$ only in the sense that it must not have length weakly between that of the two exchanging rods, covering relations in mapping $[\mu, \nu]$ down to the $m$-core poset are preserved. Since rod $d$ is fixed in every transposition sequence from $\mu$ to $\nu$, the map from $\lambda$ to $\lambda^{(d)}$ is surjective on the interval $\left[\mu^{(d)}, \nu^{(d)}\right]$.

Conversely, given any $m$-core $\gamma \in\left[\mu^{(d)}, \nu^{(d)}\right]$, we can lift it to an $m+1$ core by reversing the procedure. The reverse procedure is injective and also preserves the covering relations. Hence the intervals are isomorphic.

The bijection on skew strong tableaux is obtained in the obvious way, by mapping the saturated chain

$$
S=\left(\mu=\mu_{0} \subset \mu_{1} \subset \mu_{2} \subset \cdots \subset \mu_{k}=\nu\right)
$$


to the chain

$$
S^{(d)}=\left(\mu^{(d)}=\mu_{0}^{(d)} \subset \mu_{1}^{(d)} \subset \mu_{2}^{(d)} \subset \cdots \subset \mu_{k}^{(d)}=\nu^{(d)}\right) .
$$

To see that this bijection preserves the number of $i$-ribbons, recall from Corollary 5.4 that the number of $i$-ribbons of a strong tableau is equal to the difference in length of the interchanging rods taking $\lambda_{i-1}$ to $\lambda_{i}$. Since the map from $m+1$-cores to $m$-cores preserves the relative lengths of all rods other than rod $d$, this number is clearly preserved.

By Proposition 7.4, the following map is well defined.

Definition 7.6. Let $\mu \subset \nu$ be $m+1$-cores such that some (equivalently, every) transposition sequence from $\mu$ to $\nu$ does not touch rod $d$. Define the flattening map

$$
\mathrm{fl}_{d}: \operatorname{SST}^{*}(\nu / \mu, m+1) \longrightarrow \operatorname{SST}^{*}\left(\nu^{(d)} / \mu^{(d)}, m\right)
$$

sending $S^{*} \in \operatorname{SST}^{*}(\nu / \mu, m+1)$ to the underlying strong tableau $S^{(d)}$ with the stars placed on each $i$-ribbon in such a way as to preserve the depth.

Note that the flattening map does not, in general, preserve the spin statistic because it can shorten the height of ribbons.

Proposition 7.7. Let $\mu \subset \nu$ be $m+1$-cores such that some transposition sequence from $\mu$ to $\nu$ does not touch rod $d$. The flattening map $\mathrm{fl}_{d}$ : $\operatorname{SST}^{*}(\nu / \mu, m+1) \longrightarrow \operatorname{SST}^{*}\left(\nu^{(d)} / \mu^{(d)}, m\right)$ is a bijection preserving the signature of a starred strong tableau and it commutes with the involutions $\varphi_{i}$ for all $1<i<\ell(\nu)-\ell(\mu)$.

Proof. To see $\mathrm{fl}_{d}$ preserves the signature $\sigma\left(S^{*}\right)$, recall from Definition 5.5 and Corollary 5.6 that the content of $i^{*}$ is determined by an excess bead on the longer rod in the $i$ th exchange on the $n$-rod abacus. Since the relative order among the beads on the abacus is unchanged by the procedure in Definition 7.2, the contents of $i^{*},(i+1)^{*}$ will form a decent in $\sigma\left(S^{*}\right)$ if and only if there is a corresponding descent in $\sigma\left(\mathrm{fl}_{d}\left(S^{*}\right)\right)$. This proves $\sigma\left(S^{*}\right)=$ $\sigma\left(\mathrm{fl}_{d}\left(S^{*}\right)\right)$.

To show $\mathrm{fl}_{d}\left(\varphi_{i}\left(S^{*}\right)\right)=\varphi_{i}\left(\mathrm{fl}_{d}\left(S^{*}\right)\right)$, simply note that the cases in the definition of $\varphi_{i}$ depend only on the types of rod exchanges in the corresponding 3 -interval of the $m+1$ or $m$-core poset respectively. But, the relative order among the endpoints of the exchanging rods and the isomorphism type of the interval are persevered by the flattening map. Hence the flattening map and the involution commute. 
Corollary 7.8. Let $\mu \subset \nu$ be $n$-cores with $\nu$ lying $r$ ranks above $\mu$. If $2 r<n$, then for $m=2 r$, there exists $m$-cores $\widehat{\mu} \subset \widehat{\nu}$ such that there exists a bijection from $\operatorname{SST}^{*}(\nu / \mu, n)$ to $\operatorname{SST}^{*}(\widehat{\nu} / \widehat{\mu}, m)$ that preserves the signature and commutes with the involutions $\varphi_{i}$ for all $1<i<\ell(\nu)-\ell(\mu)$. Thus, the affine dual equivalence graphs of $\widehat{\nu} / \widehat{\mu}$ and $\nu / \mu$ are isomorphic as signed colored graphs.

Proof. If $\operatorname{rank}(\nu)-\operatorname{rank}(\mu)=r$, then every transposition sequence from $\mu$ to $\nu$ has exactly $r$ elements and at most $2 r$ distinct subscripts. Therefore, by Proposition 7.4, we can find an interval isomorphic to $[\mu, \nu]$ in the $m$-core poset after removing all fixed rods on this interval. This isomorphism preserves the number of $i$-ribbons for each $i$. Thus, it extends to an isomorphism from $\operatorname{SST}^{*}(\nu / \mu, n)$ to $\operatorname{SST}^{*}(\widehat{\nu} / \widehat{\mu}, m)$ by requiring the depth of the starred $i$ ribbon to be constant under this map for each $i$. The length of the $i$-ribbons and the contents of the $i$-ribbons can change under this bijection. However, the relative order among the contents of the ribbons will not change since the depth is preserved and the length differences of the remaining rods are exactly the same. We concluded that the signature is preserved under the bijection. Furthermore, the conditions for the witness and for the involutions $\varphi_{i}$ for all $1<i<\ell(\nu)-\ell(\mu)$ only depend on the relative order of the contents and lengths of consecutive ribbons. These relative orders will be unchanged by the bijection on starred strong tableaux. This allows us to conclude that the affine dual equivalence graphs of $\widehat{\nu} / \widehat{\mu}$ and $\nu / \mu$ are isomorphic as signed colored graphs.

\subsection{The cloning map}

Whereas flattening removes rows of the abacus, cloning adds columns. Analogous to flattening, we will define cloning on starred strong tableaux so that it preserves the signatures. In some cases, cloning commutes with the affine dual equivalence operators $\varphi_{i}$.

Definition 7.9. For any $n$-core $\mu$, define $\mu_{(j)}$ to be the unique partition associated to the abacus obtained by cloning the column of the $n$-rod abacus of $\mu$ containing positions $j, j+1, \ldots, j+n-1$. Specifically, let $\beta$ be the binary string encoding the abacus for $\mu$. Then $\mu_{(j)}$ is the abacus associated to the string obtained from $\beta$ by inserting a copy of the substring $\beta_{j}, \beta_{j+1}, \ldots, \beta_{j+n-1}$ into the abacus for $\mu$ between positions $j-1$ and $j$.

Cloning a column has the effect of extending some of the rods in the $n$-rod abacus, hence $\mu_{(j)}$ is also an $n$-core. To see the effect of cloning on partitions, consider taking $(5,2,2)$ regarded as a 4 -core and cloning the 
column beginning with content 0 . This gives $(5,2,2)_{(0)}=(7,4,4,2,2)$, as depicted below.
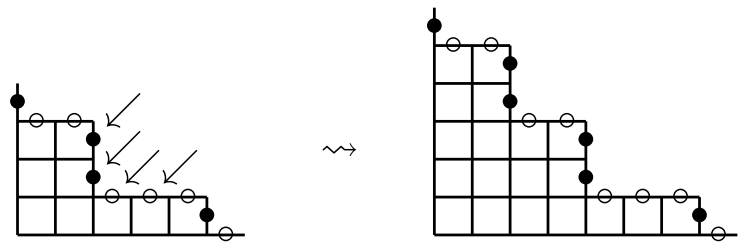

Observe that for fixed $\mu$, different values for $j$ can lead to the same $n$ core $\mu_{(j)}$. For instance, taking any $j \in\{-4, \ldots, 0\}$ results in $(5,2,2)_{(j)}=$ $(7,4,4,2,2)$.

In order for flattening to preserve a covering relation in the $n$-core poset, the transposition sequence simply needs to avoid the rod being removed. The situation for cloning is more subtle. Covering relations are not always preserved even when the replicated column is disjoint from the indexing transposition. It is immediate from Proposition 5.3 that if $t_{r, s} \mu>\mu$ is a covering relation in the $n$-core poset, then $\left(t_{r, s} \mu\right)_{(j)}$ covers $\mu_{(j)}$ in the $n$-core poset if and and only if for every $r<h \leq s$ the relative order of the lengths of rods $h, r$ and $s$ is the same in both $\mu$ and $\mu_{(j)}$. We call such a $j$ a cloneable index for $\mu \subset t_{r, s} \mu$. More generally, $j$ is a cloneable index for the interval $[\mu, \nu]$ provided cloning the column beginning at $j$ of every core partition in the interval results in another isomorphic interval in the $n$-core poset. This happens if and only if no rod in the n-rod abacus representing any element in the interval has a rightmost bead of content $j, j+1, \ldots, j+n-1$. Similarly, we say $j$ is a cloneable index for $S^{*} \in \operatorname{SST}^{*}(\nu / \mu, n)$ provided $j$ is a cloneable index for $[\mu, \nu]$. The clone of $S^{*}$, denoted $\operatorname{cl}_{j}\left(S^{*}\right)$, is defined to be the saturated chain obtained from $S$ by cloning, the column beginning with $j$ in each $n$-rod abacus in the chain and leaving all the stars with content less than $j$ at the same depth and increasing the depth by 1 for all stars with content at least $j$. Note, all $i$-ribbons will have the same shape in $S^{*}$ and $\operatorname{cl}_{j}\left(S^{*}\right)$ since the relative order of the rod lengths is unchanged by cloning a column. See Figure 13 for example.

Observe that if $S^{*} \in \operatorname{SST}^{*}(\nu / \mu, n)$ and $j$ is a cloneable index for $S^{*}$, then $j$ is a cloneable index for every other starred strong tableau in $\operatorname{SST}^{*}(\nu / \mu, n)$ as well since the definition of a cloneable index only depends on the interval $[\mu, \nu]$.

Definition 7.10. Assume that $S^{*}$ has a cloneable index at $j$ and that $T^{*}=\operatorname{cl}_{j}\left(S^{*}\right) \in \operatorname{SST}^{*}(\beta / \alpha, n)$. Define the cloning map on components

$$
\operatorname{cl}_{j}:\left[S^{*}\right] \longrightarrow \operatorname{SST}^{*}(\beta / \alpha, n)
$$


3
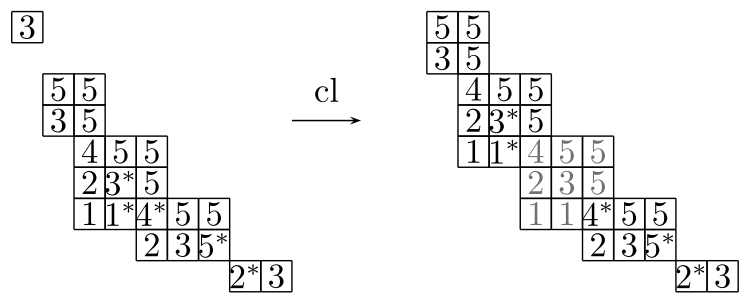

Figure 13: An example of the cloning map on a starred strong tableau. The cloned cells are red in the electronic version.

by cloning each starred strong tableaux in $\left[S^{*}\right]$ at the column beginning with $j$. The inverse map to cloning, when it is defined, will be denoted by

$$
\mathrm{sq}_{j}:\left[T^{*}\right] \longrightarrow\left[S^{*}\right]
$$

and we call it the squashing map.

As with the flattening map, the cloning map does not, in general, preserve the spin statistic since it may alter the number of $i$-ribbons and/or it may alter the depth of the starred ribbons. Nonetheless, once the cloning map commutes with the $\varphi_{i}$ 's on a connected component of an affine dual equivalence graph then we can clone the same column any number of times and get an isomorphic component.

The following proposition is the analog of Proposition 7.7.

Proposition 7.11. Assume that $S^{*} \in \operatorname{SST}^{*}(\nu / \mu, n)$ has a cloneable index at $j$ and that $T^{*}=\operatorname{cl}_{j}\left(S^{*}\right)$. Further assume that cloning the column beginning at $j$ commutes with the involutions $\varphi_{i}$ for all $1<i<\ell(\nu)-\ell(\mu)$ on the component $\left[S^{*}\right]$. Then $j$ is a cloneable index for every starred strong tableaux in $\left[T^{*}\right]$. Moreover, if $U^{*}=\operatorname{cl}_{j}\left(T^{*}\right)$, then $\mathrm{cl}_{j}:\left[T^{*}\right] \longrightarrow\left[U^{*}\right]$ is a bijection preserving the signature of each starred strong tableau and it commutes with the involutions $\varphi_{i}$ for all $1<i<\ell(\nu)-\ell(\mu)$. Thus, $\left[S^{*}\right] \approx\left[T^{*}\right] \approx\left[U^{*}\right]$ as signed, colored graphs.

Proof. The fact that $j$ is again a cloneable index for $T^{*}$ follows directly from the characterization of $j$ being a cloneable index for the interval containing $S^{*}$ in terms of rod lengths.

To see that $\left[T^{*}\right]$ is isomorphic to $\left[U^{*}\right]$ as signed colored graphs, one must check that the affine dual equivalence maps $\varphi_{i}$ commute with the cloning map from $\left[T^{*}\right]$ to $\left[U^{*}\right]$. This follows since the conditions for the 
affine dual equivalence map on rank 3 intervals are unchanged at each step by removing $n$ consecutive content diagonals that contains no head or tail of a starred ribbon in any of the starred strong tableaux in $\left[U^{*}\right]$ and that the cells in those $n$ diagonals must necessarily be a copy of the next $n$-translate down.

Remark 7.12. As a consequence of Propositions 7.7 and 7.11, we observe that the process of flattening and squashing a component in an affine dual equivalence graph as much as possible is similar to applying the necessary jeu de taquin slides which bring together all of the connected components in a skew tableaux by removing empty rows and columns. Note, both flattening and cloning/squashing can change the spin statistic even when they commute with affine dual equivalence on a component. Thus a complete analog of jeu de taquin generalizing these moves would need to keep track of powers of $t$ separately from the algorithm.

\subsection{Local Schur positivity}

Our next goal is to show that there are only a small number of isomorphism classes of connected components of rank 4 affine dual equivalence graphs. Recall that a starred strong tableau $S^{*}$ on an interval $[\mu, \nu]$ has ribbons labeled $1,2, \ldots, \ell(\nu)-\ell(\mu)$. We say $S^{*}$ has rank $r$ provided $r=\ell(\nu)-\ell(\mu)$. The component $\left[S^{*}\right]$ of the affine dual equivalence graph on $\nu / \mu$ has edges labeled $2,3, \ldots, r-1$ and each vertex has a signature of length $r-1$.

Lemma 7.13. Let $S^{*} \in \operatorname{SST}^{*}(\nu / \mu, n)$ be a starred strong tableau of rank $k=4$. Then $\left[S^{*}\right]$ has a Schur positive generating function. In fact, each such $\left[S^{*}\right]$ is either an isolated vertex, or a path with either 2 or 4 edges with alternating color labels. See Figure 14.

This lemma can be proved in two ways. One approach is to do a computer verification by identifying a set of dual equivalence classes which contain all possible isomorphism types after flattening and squashing as much as possible. The lisp code used for this approach can be found in

http://www. math. washington.edu/ billey/kschur/verifier.faster.lisp.

The second approach is based on the reading words of the starred strong tableaux, see [3].

Remark 7.14. An extensive computer exploration for all possible isomorphism types for affine dual equivalence graphs of rank 5 was undertaken. As of November of 2011, we have observed 326 distinct isomorphism types 

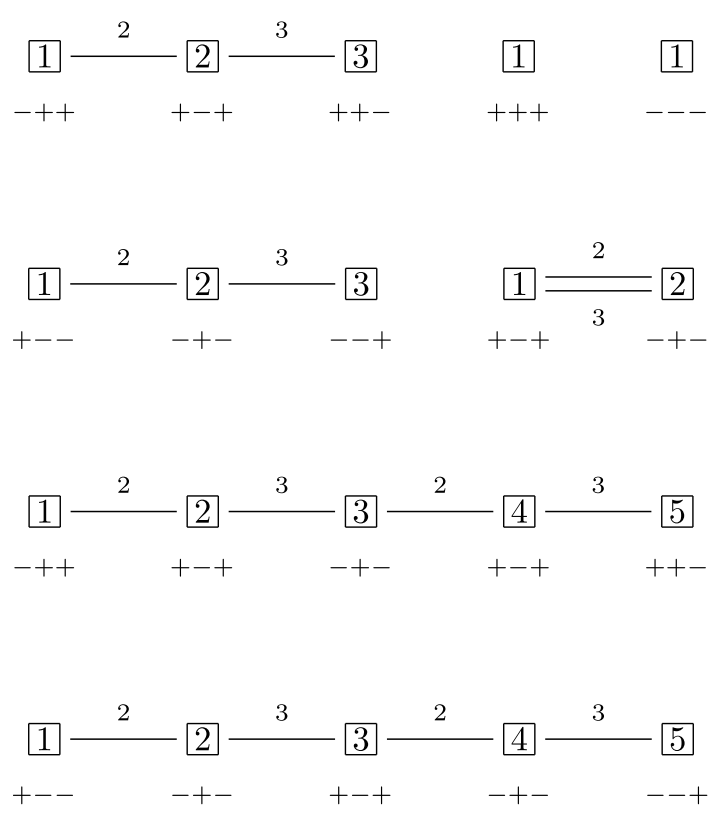

Figure 14: All 7 possible isomorphism types of connected components of affine dual equivalence graphs of rank 4.

which can be viewed in

http://www.math. washington. edu/ billey/kschur/d-graphs-11-2011.pdf.

Note for comparison, there are only 25 isomorphism types for rank 5 graphs for LLT polynomials as defined in Section 8.

Theorem 7.15. For any pair of $n$-core partitions $\mu \subset \nu$, the affine dual equivalence graph $\mathcal{G}_{\nu / \mu}^{(n)}$ is a D graph which is locally Schur positive for 2colored edges and for which spin is constant on connected components.

Proof. By Proposition 6.14, the involutions $\varphi_{i}$ preserve the spin statistic, hence spin is constant on connected components of $\mathcal{G}_{\nu / \mu}^{(n)}$.

To prove $\mathcal{G}_{\nu / \mu}^{(n)}$ is a $\mathrm{D}$ graph, we must verify the axioms in Definition 4.5. Axiom 1 follows from Theorem 6.12 where $\varphi_{i}$ is shown to be an involution which switches the sign appropriately. Axioms 2 and 5 follow from the fact that $\varphi_{i}$ affects only $i-1, i$ and $i+1$-ribbons. Axiom 3 and the $\mathrm{LSP}_{2}$ property both follow from Lemma 7.13 since every connected component of $\mathcal{G}_{\nu / \mu}^{(n)}$ restricted to $E_{i-1} \cup E_{i}$ is isomorphic to a component of a rank 4 affine dual equivalence graph replacing the edge labels 2,3 by $i-1, i$ respectively. 
Note that affine dual equivalence graphs need not satisfy Axiom 4 of Definition 4.2. It is not known if affine dual equivalence graphs satisfy Axiom 6 .

\section{Connections with LLT, Macdonald polynomials and Beyond}

The primary interest in $k$-Schur functions originally was the conjecture of Lapointe, Lascoux and Morse that these functions straddle the gap between Macdonald polynomials and Schur functions. That is, when $\mu$ is a $k$-bounded partition, they conjecture

$$
H_{\mu}(X ; q, t)=\sum_{\nu \leq \mu} K_{\nu, \mu}^{(k)}(q, t) s_{\nu}^{(k)}(X ; t),
$$

where $K_{\nu, \mu}^{(k)}(q, t) \in \mathbb{N}[q, t]$, and

$$
s_{\nu}^{(k)}(X ; t)=\sum_{\lambda \leq \nu} C_{\lambda, \nu}^{(k)}(t) s_{\lambda}(X),
$$

where $C_{\lambda, \nu}^{(k)}(t) \in \mathbb{N}[t]$.

The Macdonald polynomials, the LLT polynomials, and the $k$-Schur functions using the definition given in this paper, expand positively in the basis of fundamental quasisymmetric functions with indexing sets based on generalized tableaux. See [10] for details on the Macdonald and LLT expansions. We now have a signed colored graph structure on the generalized tableaux in each case by the construction in [2] and Theorem 7.15. It is an open problem to describe how these graphs relate to each other. Toward finding such a connection, we have identified a $\mathrm{D}$ graph isomorphism $\theta$ between the graph of starred strong tableaux on the 2 -core $(m, m-1, \ldots, 1)$ and the graph of standard filling of the $m$-tuple $((1), \ldots,(1))$ each embedded at content 0 . Furthermore, $\left(\begin{array}{c}m \\ 2\end{array}\right)-\operatorname{spin}\left(S^{*}\right)=\operatorname{inv}\left(\theta\left(S^{*}\right)\right)$.

In fact, for any family of functions expressible in terms of fundamental quasisymmetric functions which are conjectured to be Schur positive, one can look for a signed-colored graph structure or analog of dual equivalence on the indexing set which refines the Schur positivity question to connected components. Another example of this phenomena arises in the work of Loehr and Warrington [25]. They showed that the plethysm of two Schur functions has a nice expansion in terms of fundamental quasisymmetric functions indexed by a family of objects in analogy with standard tableaux. Is there a 


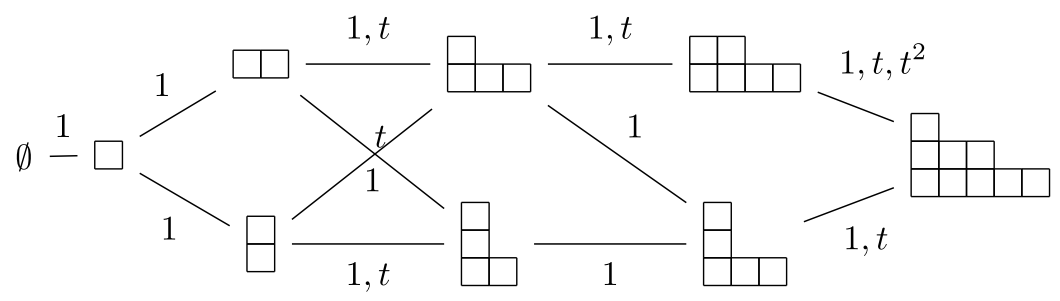

Figure 15: The poset of 3-cores lying below $(5,3,1)$, with edge weights giving the spin contributions of possible starrings.

nice signed-colored graph structure for these objects? Also, the Stanley symmetric functions are indexed by reduced expressions for permutations and expand positively in terms of both fundamental quasisymmetric functions and Schur functions. Is there a family of standard objects and an analog of a dual equivalence graph that goes with the Stanley symmetric functions?

\section{Appendix A. Examples}

In this appendix we give the quasisymmetric and Schur expansion for the $k$-Schur function $s_{(2,2,1)}^{(2)}$. We compute this using the interval $[\emptyset,(5,3,1)]$ of the 3 -core poset (Figure 15) and the corresponding D graph on all starred strong tableaux of shape $(5,3,1)$ regarded as a 3 -core (Figure 16).

Thus, we obtain from Figure 16 that

$$
\begin{aligned}
s_{(2,2,1)}^{(2)}= & Q_{-+--}+Q_{--+-}+t Q_{++--}+(1+t) Q_{+--+} \\
& +\left(2 t+t^{2}\right) Q_{-++-}+\left(1+2 t+t^{2}\right) Q_{+-+-} \\
& +\left(1+2 t+t^{2}\right) Q_{-+-+}+t Q_{--++}+\left(t^{2}+t^{3}\right) Q_{+++-} \\
& +\left(t+2 t^{2}+t^{3}\right) Q_{++-+}+\left(t+2 t^{2}+t^{3}\right) Q_{+-++} \\
& +\left(t^{2}+t^{3}\right) Q_{-+++}+t^{4} Q_{++++} \\
= & s_{(2,2,1)}+t s_{(3,1,1)}+\left(t+t^{2}\right) s_{(3,2)}+\left(t^{2}+t^{3}\right) s_{(4,1)}+t^{4} s_{(5)} .
\end{aligned}
$$

\section{Acknowledgments}

We would like to thank Nantel Bergeron, Andrew Crites, Adriano Garsia, Jim Haglund, Mark Haiman, Steve Mitchell, Jennifer Morse, Austin Roberts and Mike Zabrocki for inspiring conversations on this topic, and an anonymous referee for many useful comments. 


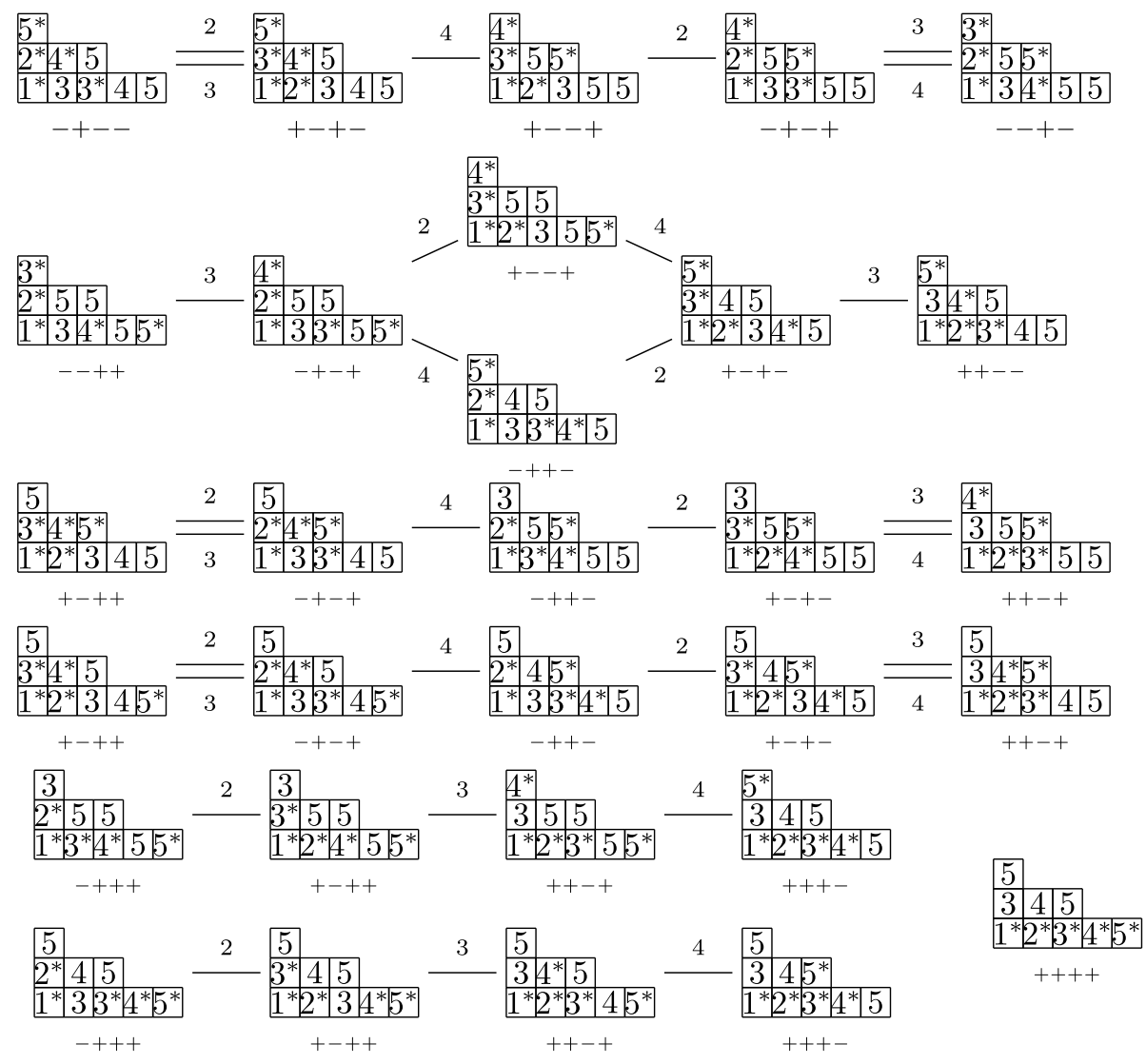

Figure 16: The D graph on starred strong tableaux of shape $(5,3,1)$ regarded as a 3 -core.

\section{References}

[1] S. Assaf (2007). The Schur expansion of Macdonald polynomials. Preprint. MR2710706

[2] S. Assaf. Dual equivalence graphs I: A combinatorial proof of LLT and Macdonald positivity. arXiv:1005.3759, originally 2008, revised 2011. MR2721449

[3] S. H. Assaf. On dual equivalence and Schur positivity. ArXiv e-prints, June 2011.

[4] C. Berg, B. Jones, and M. Vazirani (2009). A bijection on core partitions 
and a parabolic quotient of the affine symmetric group. J. Combin. Theory Ser. A 116(8) 1344-1360. MR2568803

[5] A. Björner and F. Brenti (1996). Affine permutations of type A. Electron. J. Combin. 3(2), Research Paper 18, approx. 35 pp. (electronic). The Foata Festschrift. MR1392503

[6] W. Fulton (1997). Young Tableaux; With Applications To Representation Theory And Geometry, volume 35 of London Mathematical Society Student Texts. Cambridge University Press, New York. MR1464693

[7] A. M. Garsia and M. Haiman (1993). A graded representation model for Macdonald's polynomials. Proc. Natl. Acad. Sci. USA 90(8) 36073610. MR1214091

[8] I. M. Gessel (1984). Multipartite $P$-partitions and inner products of skew Schur functions. In: Combinatorics and Algebra (Boulder, Colo., 1983), Vol. 34, Contemp. Math., Amer. Math. Soc., Providence, RI, pp. 289-317. MR0777705

[9] J. Haglund (2004). A combinatorial model for the Macdonald polynomials. Proc. Natl. Acad. Sci. USA 101(46) 16127-16131 (electronic). MR2114585

[10] J. Haglund, M. Haiman, and N. Loehr (2005). A combinatorial formula for Macdonald polynomials. J. Amer. Math. Soc. 18(3) 735-761 (electronic). MR2138143

[11] M. Haiman (2001). Hilbert schemes, polygraphs and the Macdonald positivity conjecture. J. Amer. Math. Soc. 14(4) 941-1006 (electronic). MR1839919

[12] M. D. Haiman (1992). Dual equivalence with applications, including a conjecture of Proctor. Discrete Math. 99(1-3) 79-113. MR1158783

[13] C. R. H. Hanusa and B. C. Jones. Abacus models for parabolic quotients of affine Weyl groups. arXiv:1105.5333, May 2011. MR2921615

[14] G. James and A. Kerber. The representation theory of the symmetric group, Vol. 16, Encyclopedia of Mathematics and its Applications. Addison-Wesley Publishing Co., Reading, Mass., 1981. With a foreword by P. M. Cohn; With an introduction by Gilbert de B. Robinson. MR0644144

[15] T. Lam (2008). Schubert polynomials for the affine Grassmannian. J. Amer. Math. Soc. 21(1) 259-281 (electronic). MR2350056 
[16] T. Lam, L. Lapointe, J. Morse, and M. Shimozono. k-shape poset and branching of $k$-Schur functions. Preprint, 2010. arXiv:1007.5334v1. MR2741963

[17] T. Lam, L. Lapointe, J. Morse, and M. Shimozono (2010). Affine insertion and Pieri rules for the affine Grassmannian. Mem. Amer. Math. Soc. 208(977). MR2741963

[18] L. Lapointe, A. Lascoux, and J. Morse (2003). Tableau atoms and a new Macdonald positivity conjecture. Duke Math. J. 116(1) 103-146. MR1950481

[19] L. Lapointe and J. Morse (2003). Schur function analogs for a filtration of the symmetric function space. J. Combin. Theory Ser. A 101(2) 191-224. MR1961543

[20] L. Lapointe and J. Morse (2003). Schur function identities, their $t$-analogs, and $k$-Schur irreducibility. Adv. Math. 180(1)222-247. MR2019223

[21] L. Lapointe and J. Morse (2005). Tableaux on $k+1$-cores, reduced words for affine permutations, and $k$-Schur expansions. J. Combin. Theory Ser. A 112(1)44-81. MR2167475

[22] L. Lapointe and J. Morse (2007). A $k$-tableau characterization of $k$ Schur functions. Adv. Math. 213(1) 183-204. MR2331242

[23] A. Lascoux (2001). Ordering the affine symmetric group. In Algebraic Combinatorics and Applications (Gößweinstein, 1999), Springer, Berlin, pp. 219-231. MR1851953

[24] A. Lascoux, B. Leclerc, and J.-Y. Thibon (1997). Ribbon tableaux, Hall-Littlewood functions, quantum affine algebras, and unipotent varieties. J. Math. Phys. 38(2) 1041-1068. MR1434225

[25] N. A. Loehr and G. S. Warrington (2012). Quasisymmetric expansions of Schur-function plethysms. Proc. Amer. Math. Soc. 140(4) 11591171. MR2869102

[26] I. G. Macdonald (1988). A new class of symmetric functions. Actes du 20e Seminaire Lotharingien 372 131-171.

[27] I. G. Macdonald (1995). Symmetric Functions and Hall Polynomials, 2nd ed. Oxford Mathematical Monographs. The Clarendon Press Oxford University Press, New York. With contributions by A. Zelevinsky, Oxford Science Publications. MR1354144 
[28] K. Misra and T. Miwa (1990). Crystal base for the basic representation of $U_{q}(s l(n))$. Comm. Math. Phys. 134(1) 79-88. MR1079801

[29] R. P. Stanley (1997). Enumerative Combinatorics. Vol. 1, Vol. 49 Cambridge Studies in Advanced Mathematics. Cambridge University Press, Cambridge. With a foreword by Gian-Carlo Rota; Corrected reprint of the 1986 original. MR1090542

Sami H. Assaf

Department of Mathematics

University of Southern CALIFornia

3620 S Vermont Ave KAP 104

Los ANgeles, CA 90089-2532

USA

E-mail address: shassaf@usc.edu

Sara C. Billey

Mathematics Department

UNIVERSITY OF WASHINGTON

Box 354350

SEATtLe, WA 98105

USA

E-mail address: billey@uw.edu

RECEIVED JANUARY 6, 2012 